Accepted for publication in The Astrophysical Journal

Preprint typeset using $\mathrm{LATE}_{\mathrm{E}} \mathrm{X}$ style emulateapj v. 04/17/13

\title{
HUBBLE FRONTIER FIELDS FIRST COMPLETE CLUSTER DATA: FAINT GALAXIES AT $z \sim 5-10$ FOR UV LUMINOSITY FUNCTIONS AND COSMIC REIONIZATION
}

\author{
Masafumi Ishigaki $^{1,2}$, Ryota Kawamata ${ }^{3}$, Masami Ouchi ${ }^{1,4}$, Masamune OGuri ${ }^{2,4,5}$, \\ Kazuhiro Shimasaku ${ }^{3,5}$, AND Yoshiaki ONO ${ }^{1}$ \\ Accepted for publication in The Astrophysical Journal
}

\begin{abstract}
We present the comprehensive analyses of faint dropout galaxies up to $z \sim 10$ with the first full-depth data set of Abell 2744 lensing cluster and parallel fields observed by the Hubble Frontier Fields (HFF) program. We identify 54 dropouts at $z \sim 5-10$ in the HFF fields, and enlarge the size of $z \sim 9$ galaxy sample obtained to date. Although the number of highly magnified $(\mu \sim 10)$ galaxies is small due to the tiny survey volume of strong lensing, our study reaches the galaxies' intrinsic luminosities comparable to the deepest-field HUDF studies. We derive UV luminosity functions with these faint dropouts, carefully evaluating the combination of observational incompleteness and lensing effects in the image plane by intensive simulations including magnification, distortion, and multiplication of images, with the evaluations of mass model dependences. Our results confirm that the faint-end slope, $\alpha$, is as steep as -2 at $z \sim 6-8$, and strengthen the evidence of the rapid decrease of UV luminosity densities, $\rho_{\mathrm{UV}}$, at $z>8$ from the large $z \sim 9$ sample. We examine whether the rapid $\rho_{\mathrm{UV}}$ decrease trend can reconcile with the large Thomson scattering optical depth, $\tau_{\mathrm{e}}$, measured by CMB experiments allowing a large space of free parameters such as average ionizing photon escape fraction and stellar-population dependent conversion factor. No parameter set can reproduce both the rapid $\rho_{\mathrm{UV}}$ decrease and the large $\tau_{\mathrm{e}}$. It is possible that the $\rho_{\mathrm{UV}}$ decrease moderates at $z \gtrsim 11$, that the free parameters significantly evolve towards high- $z$, or that there exist additional sources of reionization such as X-ray binaries and faint AGNs.
\end{abstract}

Keywords: dark ages, reionization, first stars - galaxies: formation - galaxies: evolution - galaxies: high-redshift - gravitational lensing: strong

\section{INTRODUCTION}

Cosmic reionization history and sources of reionization are open questions in astronomy today. Studies of QSO Gunn-Peterson absorption indicate that the intergalactic medium (IGM) is rapidly ionized at $z \sim 6$ (Fan et al. 2006). A moderately large neutral hydrogen fraction at $z \gtrsim 6$ is implied by the $\operatorname{Ly} \alpha$ damping wing absorption features in the spectra of gamma-ray bursts (GRBs) at $z \sim 6$ (Totani et al. 2006, 2013) and $\operatorname{Ly} \alpha$ emitters at $z \sim 6-7$ (Kashikawa et al. 2006; Ouchi et al. 2010. Kashikawa et al. 2011; Konno et al. 2014). Similarly, there are reports of Ly $\alpha$-emitting galaxy fraction drops at $z \sim 7$ probably due to the increase of Ly $\alpha$ damping wing absorption given by the neutral hydrogen in the IGM (Pentericci et al. 2011, 2014, Ono et al. 2012 Schenker et al. 2012, 2014; Treu et al. 2013, Finkelstein et al. 2013). Recent observations of cosmic microwave background (CMB) present the large value of the Thomson scattering optical depth $\tau_{e}=0.091_{-0.014}^{+0.013}$ (Planck Collaboration et al. 2013). The large value of $\frac{\tau_{e}}{\text { indi- }}$

\footnotetext{
ishigaki@icrr.u-tokyo.ac.jp

i Institute for Cosmic Ray Research, The University of Tokyo, Kashiwa, Chiba 277-8582, Japan

2 Department of Physics, University of Tokyo, 7-3-1 Hongo, Bunkyo-ku, Tokyo 113-0033, Japan

${ }^{3}$ Department of Astronomy, University of Tokyo, 7-3-1 Hongo, Bunkyo-ku, Tokyo 113-0033, Japan

${ }^{4}$ Kavli Institute for the Physics and Mathematics of the Universe (Kavli IPMU, WPI), University of Tokyo, Kashiwa, Chiba 277-8583, Japan

${ }^{5}$ Research Center for the Early Universe, University of Tokyo, 7-3-1 Hongo, Bunkyo-ku, Tokyo 113-0033, Japan
}

cates that the reionization takes place at $z=11.1 \pm 1.1$ if an instantaneous reionization is assumed. The combination of these data implies that the reionization process is extended at $z \sim 6-11$.

Star-forming galaxies are thought to be major sources of the cosmic reionization (see reviews of Fan et al. 2006 Robertson et al. 2010). Recent ultra-deep observations with the Wide Field Camera 3 (WFC3) aboard the $\mathrm{Hub}$ ble Space Telescope (HST) have provided improved estimates of the abundances of star-forming galaxies at $z \sim 7-10$ (Ellis et al. 2013; Schenker et al. 2013 McLure et al. 2013). Combining these results with the WMAP constraints on the Thomson scattering optical depth (Hinshaw et al. 2013) and stellar mass densities, Robertson et al. (2013) suggest that all these observations can be explained consistently if their population of star-forming galaxies extends below the survey limits down to absolute UV magnitudes of $M_{\mathrm{UV}} \sim-13$. However, it is difficult to translate the UV luminosity function measurements into the ionized hydrogen fraction, because of uncertainties of the following three unknown parameters. The first is the escape fraction $f_{\text {esc }}$, which is the fraction of the numbers of ionizing photons escaping into the IGM to those produced by star-formation in a galaxy. The second is the conversion factor $\xi_{\text {ion }}$, which converts a UV luminosity density to the ionizing photon emission rate in a star-forming galaxy. The third is a clumping factor $C_{\mathrm{H}_{\mathrm{II}}} \equiv\left\langle n_{\mathrm{H}_{\mathrm{II}}}^{2}\right\rangle /\left\langle n_{\mathrm{H}_{\mathrm{II}}}\right\rangle^{2}$, where $n_{\mathrm{H}_{\mathrm{II}}}$ are the local number density of ionized hydrogen and the brackets indicate spatial average. It is critically important to take into account the uncertainties of these 
parameters to estimate the contribution of galaxies to reionization.

Moreover, the abundance of faint galaxies at high redshift is unknown. Some theoretical studies indicate that the star formation is suppressed in low-mass halos. Boylan-Kolchin et al. (2014) suggest that the star formation is suppressed in halos smaller than $\sim 10^{9} M_{\odot}$ at high redshift, corresponding to $M_{\mathrm{UV}} \simeq-14$. Cosmological hydrodynamical simulations of Jaacks et al. (2013) exhibit a turnover of the $z=8 \mathrm{UV}$ luminosity function at $M_{\mathrm{UV}} \sim-17$. Thus it is not obvious if the UV luminosity function of star-forming galaxies indeed extends down to $M_{\mathrm{UV}} \sim-13$, as assumed in Robertson et al. (2013). Recent observations of nearby dwarf galaxies find that the star formation in dwarf galaxies is suppressed at the epoch of reionization (Benitez-Llambay et al. 2014; Weisz et al. 2014). The faint-end slope $\alpha$ of the UV luminosity function is also not well known at high redshift. The steepening of UV luminosity functions towards high- $z$ is a general agreement of observational studies. Bouwens et al. (2014) conclude that the value of $\alpha$ evolves from $\alpha \sim-1.6$ at $z \sim 4$ to $\alpha \sim-2.0$ at $z \sim 7$. However, the determination of $\alpha$ includes a large uncertainty at $z \gtrsim 9$, due to the poor statistics of the $z \gtrsim 9$ luminosity function measurement.

Gravitational lensing by massive clusters is an effective tool to reveal properties of faint galaxies at high redshift. Lensing magnifications of background sources enable us to observe intrinsically faint sources that are not detected without lensing magnifications. For example, Cluster Lensing And Supernova survey with Hubble (CLASH) studies properties of faint star-forming galaxies at $z \sim 6-9$ using the lensing technique (Bouwens et al. 2012, Bradley et al. 2013). Recently, HST has started revolutionary deep imaging on the six massive clusters with parallel observations, the Hubble Frontier Fields (HFF; PI: J. Lotz) project whose data are $\sim 1 \mathrm{mag}$ deeper than those of CLASH. The HFF project identifies faint sources reaching $\sim 29 \mathrm{AB}$ mag, allowing us to detect background sources with intrinsic magnitudes of $\gtrsim 30$ mag by lensing magnification (Coe et al. 2014). The HFF first targets the Abell 2744 cluster, followed by other five clusters: MACSJ0416.1-2403, MACSJ0717.5+3745, MACSJ1149.5+2223, Abell S1063 (RXCJ2248.7-4431), and Abell 370. The observations of Abell 2744 were just completed in July 2014, which provide the first full-depth data set on an HFF target.

In this paper, we identify star-forming galaxies at $z \sim$ 5-10 magnified by gravitational lensing in the Abell 2744 cluster and its parallel fields. We refer to the former as the cluster field and the latter as the parallel field in the remainder of this paper. This work serves as a precursor study that uses the first one sixth of the full-depth HFF data set. We construct the mass model of Abell 2744, and derive the UV luminosity functions with the star-forming galaxies at $z \sim 5-10$. Calculating the UV luminosity densities from the UV luminosity functions of our and previous studies, we discuss cosmic reionization based on the UV luminosity density measurements and Thomson scattering optical depths from CMB observations with the ionization equation that allows a large free parameter space.

We present details of the observational data in Section
2. The photometric catalog and dropout selection methods are described in Section 3, and our mass model of Abell 2744 is presented in Section 4. Using these data, we derive the parameters of UV luminosity functions in Section 5. In Section 6, we discuss cosmic reionization with the UV luminosity densities and Thomson scattering optical depths. Finally, we summarize our results in Section 7. We adopt a cosmology with $\Omega_{\mathrm{m}}=0.3$, $\Omega_{\Lambda}=0.7, \Omega_{\mathrm{b}}=0.04$, and $H_{0}=70 \mathrm{~km} \mathrm{~s}^{-1} \mathrm{Mpc}^{-1}$.

\section{DATA}

The Abell 2744 cluster and the parallel fields were observed with WFC3-IR and Advanced Camera for Survey $(\mathrm{ACS})$ in the HFF project. These data were reduced and released to the public through the HFF official website. ${ }^{6}$ They provide drizzled science images and inverse variance weight images in four WFC3-IR bands, F105W $\left(Y_{105}\right), \mathrm{F} 125 \mathrm{~W}\left(J_{125}\right)$, F140W $\left(J H_{140}\right)$, and F160W $\left(H_{160}\right)$, and in three ACS bands, F435W $\left(B_{435}\right), \mathrm{F} 606 \mathrm{~W}\left(V_{606}\right)$, and F814W $\left(i_{814}\right)$. We use version 1.0 of the public images with a pixel scale of $0 .{ }^{\prime} 03$ pixel $^{-1}$. For the measurements of object colors, we homogenize the point spread functions (PSFs) of the WFC3 images with IRAF (Tody 1986, 1993) imfilter package. A summary of the HST data is shown in Table 1. We measure limiting magnitudes in a $0^{\prime \prime} 4$-diameter circular aperture using SDFRED (Yagi et al. 2002, Ouchi et al. 2004). We find that the $5 \sigma$ limits are $28.5-29.1 \mathrm{mag}$ in the cluster field and $28.6-29.2 \mathrm{mag}$ in the parallel field. The cluster field contains the bright intracluster light (Montes \& Trujillo 2014), which makes the depths of the cluster field shallower than those of the parallel field.

The $5 \sigma$ limiting magnitudes presented in Table 1 are measured in the entire field. However, the intracluster light is brighter in the cluster center than in the outskirts, which causes spatial variations of the depth in the HST images. To evaluate the spatial variations, we measure the limiting magnitudes in each of $4 \times 4$ grid cells defined in Figure 1 . We present the $H_{160}$-band limiting magnitudes in the cells in Figure 1. In Figure 1, we find that the depth in the third-row second-column cell (28.61 $\mathrm{mag}$ ) is about $0.5 \mathrm{mag}$ shallower than those of the cluster outskirts $(\sim 29 \mathrm{mag})$ due to the bright intracluster light. The peak-to-peak magnitudes of spatial variations of the depths are also $\sim 0.5 \mathrm{mag}$ in the rest of WFC3-IR images and the ACS data. In the cluster field, we adopt the space-dependent limiting magnitudes as illustrated in Figure 1. For sources in the outside of the cell, we apply a limiting magnitude of the nearest cell.

\section{SAMPLES}

In this section, we select the $i-, Y$-, and $Y J$-dropout candidates in the cluster and the parallel fields with the color criteria from our source catalogs. We also compare our dropout samples with those obtained in previous studies.

\subsection{Photometric catalog}

Using SWARP (Bertin et al. 2002), we make two detection images that are co-added data of $\left(J_{125}+J_{140}+\right.$

\footnotetext{
${ }^{6}$ http://archive.stsci.edu/pub/hlsp/frontier/abell2744/ images/hst/v1.0/
} 
Table 1

Summary of the HFF Abell 2744 Data

\begin{tabular}{|c|c|c|c|}
\hline Filter & Orbits & $\begin{array}{c}\text { Detection Limits }^{\mathrm{a}} \\
5 \sigma\end{array}$ & $\begin{array}{l}\text { PSF FWHM } \\
\text { arcsec }\end{array}$ \\
\hline \multicolumn{4}{|l|}{ Cluster Field } \\
\hline $\begin{array}{l}B_{435} \\
V_{606} \\
i_{814} \\
Y_{105} \\
J_{125} \\
J H_{140} \\
H_{160} \\
J_{125}+J H_{140}+H_{160}{ }^{\mathrm{c}} \\
J H_{140}+H_{160}^{\mathrm{d}}\end{array}$ & $\begin{array}{c}24 \\
15 \\
49 \\
26 \\
13.5 \\
10 \\
27 \\
\ldots \\
\ldots\end{array}$ & $\begin{array}{c}28.51 \\
28.67 \\
28.72(28.93)^{\mathrm{b}} \\
29.05(29.03)^{\mathrm{b}} \\
28.67(28.72)^{\mathrm{b}} \\
28.74(28.74)^{\mathrm{b}} \\
28.75(28.75)^{\mathrm{b}} \\
28.92 \\
28.79\end{array}$ & $\begin{array}{c}0.10 \\
0.09 \\
0.09(0.18)^{\mathrm{b}} \\
0.16(0.18)^{\mathrm{b}} \\
0.17(0.18)^{\mathrm{b}} \\
0.17(0.18)^{\mathrm{b}} \\
0.18(0.18)^{\mathrm{b}} \\
0.17 \\
0.17\end{array}$ \\
\hline \multicolumn{4}{|l|}{ Parallel Field } \\
\hline $\begin{array}{l}B_{435} \\
V_{606} \\
i_{814} \\
Y_{105} \\
J_{125} \\
J H_{140} \\
H_{160} \\
J_{125}+J H_{140}+H_{160}{ }^{\mathrm{c}} \\
J H_{140}+H_{160} \mathrm{~d}\end{array}$ & $\begin{array}{c}28 \\
16.5 \\
42.5 \\
24 \\
12 \\
10 \\
24 \\
\cdots \\
\cdots\end{array}$ & $\begin{array}{c}28.66 \\
28.98 \\
28.90(28.97)^{\mathrm{b}} \\
29.24(29.20)^{\mathrm{b}} \\
28.88(28.87)^{\mathrm{b}} \\
28.82(28.93)^{\mathrm{b}} \\
28.99(28.99)^{\mathrm{b}} \\
29.11 \\
29.03\end{array}$ & $\begin{array}{c}0.10 \\
0.10 \\
0.10(0.19)^{\mathrm{b}} \\
0.19(0.19)^{\mathrm{b}} \\
0.18(0.19)^{\mathrm{b}} \\
0.18(0.19)^{\mathrm{b}} \\
0.19(0.19)^{\mathrm{b}} \\
0.19 \\
0.19\end{array}$ \\
\hline
\end{tabular}

a Measured in a 0.' 4-diameter circular aperture.

b The values in the parenthesis correspond to the detection limits and the PSF FWHMs of PSF-homogenized images.

c The detection image for $i$ - and $Y$-dropout selections.

d The detection image for $Y J$-dropout selection.

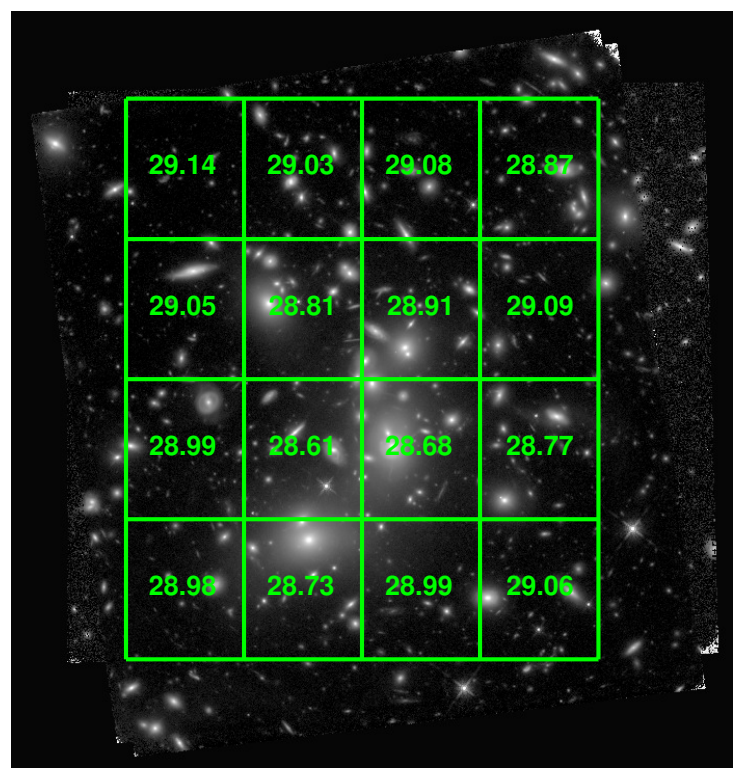

Figure 1. $H S T /$ WFC3-IR $H_{160}$ image of the Abell 2744 cluster field. The numbers denote $5 \sigma$ limiting magnitudes defined in a 0 ". 4 -diameter aperture, measured in the $4 \times 4$ grid cells.

$\left.H_{160}\right)$ and $\left(J H_{140}+H_{160}\right)$ for our $i$ - and $Y$-dropout candidates and $Y J$-dropout candidates, respectively. We match the PSFs of these band images in the same manner as the WFC3-IR images (Section 2), and produce the detection images. To apply the criteria of no bluecontinuum detections for our dropout selections, we do not match the PSFs of the blue bands whose wavelengths are shorter than the redshifted Ly $\alpha$-break feature of our dropout candidates. Because the PSF-unmatched im- ages in the blue bands provide upper limits on the flux densities that are stronger than PSF-homogenized images for point-like sources of high- $z$ galaxies, we use the PSF-unmatched data of the individual ACS images to obtain the upper limits.

We construct our source catalogs from the HFF images using SExtractor (Bertin \& Arnouts 1996) in a total of $9.5 \operatorname{arcmin}^{2}$ area where all the WFC3 and ACS images are available. We run SExtractor in dual-image mode for each set of the images. In the cluster field, we set DEBLEND_NTHRESH to 16 and DEBLEND_MINCONT to a small value of 0.0005 in order to detect objects even in highly crowded regions. In the parallel field, we use more conservative values, DEBLEND_NTHRESH $=32$ and DEBLEND_MINCONT $=0.005$, because the parallel field are not crowded. 7 The number of objects identified in the detection images is $\sim 4300$ in total. The colors of the objects are measured with magnitudes of MAG_APER $\left(m_{\mathrm{AP}}\right)$, which are estimated from the flux density within a fixed circular aperture. The aperture diameters used for $m_{\mathrm{AP}}$ are two times of the FWHMs of the PSFs. We adopt the diameters of $0.36(0.38)$ and $\sim 0$.' 2 for the PSF-matched images and for the PSF-unmatched blue-band images in the cluster (parallel) field, respectively. The detection limits are also defined with 0.36 (0".38) diameter apertures for the PSF-matched images, and $\sim 0$.' 2 diameter apertures for the PSF-unmatched images in the cluster (parallel) field.

We apply an aperture correction that is defined by the following procedure. We create a median stacked $J_{125^{-}}$ band image of our dropout candidates selected in Section 3.2 and measure the aperture flux of the stacked dropout candidate as a function of aperture size. Because the flux almost levels off at around a $1^{\prime \prime} 2$ diameter, we regard the flux within a $1^{\prime \prime}$. 2 -diameter aperture as the total flux corresponding to the total magnitude $m_{\text {tot }}$. In the stacked image, $m_{\mathrm{AP}}$ is fainter than $m_{\text {tot }}$ by $0.82 \mathrm{mag}$. We thus estimate the total magnitudes with $m_{\text {tot }}=m_{\mathrm{AP}}-c_{\mathrm{AP}}$, where $c_{\mathrm{AP}}$ is the aperture correction factor of $0.82 \mathrm{mag}$. We also make median stacked images for bright and faint subsamples of our dropout candidates, and obtain $c_{\mathrm{AP}}$ values. We confirm that the values of $c_{\mathrm{AP}}$ do not depend on luminosity beyond the statistical uncertainties in the magnitude range of our dropout candidates. Thus we apply one aperture correction factor of $c_{\mathrm{AP}}=0.82$ for all our dropout candidates.

To check the accuracy of our aperture correction, we compare $m_{\text {tot }}$ with the magnitude of MAG_AUTO $\left(m_{\text {AUTO }}\right)$, which is calculated with the Kron elliptical aperture (Kron 1980). Figure 2 presents $m_{\text {AUTO }}-m_{\text {tot }}$ as a function of $m_{\mathrm{AP}}$, and indicates that $m_{\text {tot }}$ is comparable to $m_{\text {AUTO }}$ for bright dropout candidates with $m_{\mathrm{AP}}<27$ mag. The values of $m_{\text {AUTO }}-m_{\text {tot }}$ have significant scatters at the faint magnitudes, which is mainly due to uncertainties in determining the Kron elliptical apertures of faint sources. We adopt $m_{\text {tot }}$ for our estimates of total magnitudes, as we expect that the $m_{\text {tot }}$ values are more

7 Although we use the different deblending parameter sets in the cluster and the parallel fields, this difference does not affect our final results of the UV luminosity functions. This is because we use the same deblending parameter sets, each for the cluster and parallel fields, self-consistently in our simulations to derive our UV luminosity functions (Section 5 . 


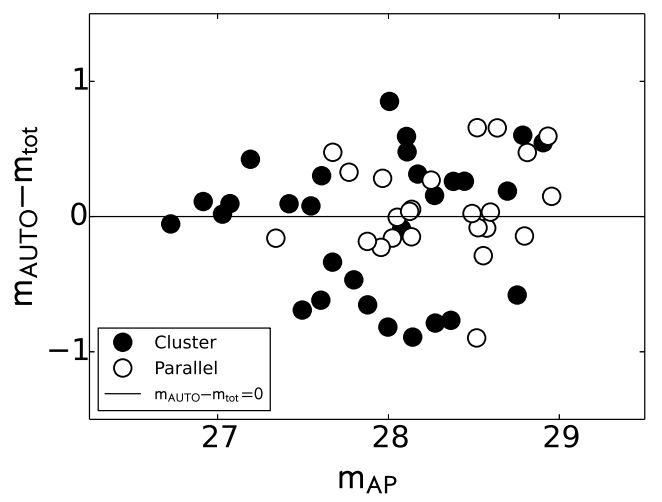

Figure 2. Difference between the aperture-corrected total magnitudes $\left(m_{\text {tot }}\right)$ and the SExtractor's AUTO magnitudes $\left(m_{\text {AUTO }}\right)$ as a function of the aperture magnitude $m_{\mathrm{AP}}$ for our dropout candidates in the cluster (filled circles) and parallel (open circles) fields

. The horizontal line corresponds to the case that $m_{\mathrm{AUTO}}$ is equal to $m_{\text {tot }}$.

reliable than $m_{\mathrm{AUTO}}$ for faint sources.

\subsection{Dropout Selection}

For the selection of $i$-dropouts at $z \sim 6-7$, we use the color criteria defined by Atek et al. (2014b):

$$
\begin{array}{r}
i_{814}-Y_{105}>0.8, \\
i_{814}-Y_{105}>0.6+2\left(Y_{105}-J_{125}\right), \\
Y_{105}-J_{125}<0.8 .
\end{array}
$$

For objects fainter than the $3 \sigma$ limiting magnitude in $i_{814}, i_{814} 3 \sigma$ upper limiting magnitude is replaced with the $i_{814}$ magnitude (see Atek et al. 2014b). For secure source detection, we apply the source identification thresholds of the $>5 \sigma$ significance levels both in the $Y_{105}$ and $J_{125}$ bands. From our $i$-dropout candidate catalog, we remove sources detected at the $>2 \sigma$ level either in the $B_{435}$ or $V_{606}$ band.

For $Y$-dropouts at $z \sim 8$, we adopt the following criteria (Schenker et al. 2013):

$$
\begin{aligned}
& Y_{105}-J_{125}>0.5, \\
& J_{125}-H_{160}<0.4 .
\end{aligned}
$$

As described in Schenker et al. (2013), we use the $1 \sigma$ upper limiting magnitude in the $\bar{Y}_{105}$ band. In this selection, sources with the $>3.5 \sigma$ levels both in the $J_{125}$ and $J H_{140}$ bands are regarded as real objects. We reject sources detected at $2 \sigma$ in the optical bands. Additionally, we apply a criterion that no more than one of the optical bands shows a detection above the $1.5 \sigma$ level. We use a collective $\chi_{\text {opt }}^{2}$ value to eliminate contamination, the details of which are described in Section 3.3 of Bouwens et al. (2011) and Section 3.2 of Schenker et al. (2013). The corrective $\chi_{\mathrm{opt}}^{2}$ is defined by $\chi_{\mathrm{opt}}^{2} \equiv \sum_{j} \operatorname{SGN}\left(f_{j}\right)\left(\mathrm{SNR}_{j}\right)^{2}$, where $f_{j}$ is the flux density in the $j$-th band, $\mathrm{SNR}_{j}$ is the signal-to-noise ratio of the source in the $j$-th band, and $\operatorname{SGN}\left(f_{j}\right)$ is a sign function; $\operatorname{SGN}\left(f_{j}\right)=1$ if $f_{j}>0$ and -1 if $f_{j}<0$. The $j$ index runs across $B_{435}, V_{606}$, and $i_{814}$. We remove objects with $\chi_{\mathrm{opt}}^{2}>5.0$ from our dropout candidates if they are brighter than the $10 \sigma$ limit in the $J H_{140}$ band, and remove ones with $\chi_{\mathrm{opt}}^{2}>2.5$ if they are fainter than the
$5 \sigma$ limit. A linear interpolation is used for objects with $J H_{140}$ between the $5 \sigma$ and $10 \sigma$ limit.

For $Y J$-dropouts at $z \sim 9$, we use the following criteria:

$$
\begin{array}{r}
\left(Y_{105}+J_{125}\right) / 2-J H_{140}>0.75, \\
\left(Y_{105}+J_{125}\right) / 2-J H_{140}> \\
0.75+0.8 \times\left(J H_{140}-H_{160}\right), \\
J_{125}-H_{160}<1.15, \\
J H_{140}-H_{160}<0.6 .
\end{array}
$$

We replace the $Y_{105}$ or $J_{125}$ magnitude with the $1 \sigma$ upper limiting magnitude if an object is fainter than the $1 \sigma$ magnitude in $Y_{105}$ or $J_{125}$, following Oesch et al. (2013). For the $Y J$-dropouts, we require detection significance levels beyond $3 \sigma$ in the $J H_{140}$ and $H_{160}$ bands, and $3.5 \sigma$ in at least one of the $J H_{140}$ and $H_{160}$ bands. From our $Y J$-dropout sample, we remove sources detected at the $2 \sigma$ level in, at least, one of the optical bands and sources with $\chi_{\mathrm{opt}}^{2}>2.8$. These criteria are similar to those defined by Oesch et al. (2013), but we slightly relax the criteria to include dropout candidates at $z \sim 9.5$.

We select $i-, Y$-, and $Y J$-dropouts with the selection criteria shown above. Figure 3 shows the two-color diagrams for our dropout candidates, together with the expected tracks of high-redshift star-forming galaxies with UV slopes of $\beta=-2$ and -3 (see Meurer et al. 1999 for the definition of $\beta$ ). Our dropout samples consist of 35 $i$-dropout, $15 Y$-dropout, and $6 Y J$-dropout candidates. These dropout candidates are listed in Tables 2-4. Figure 4 shows cutout images of our dropout candidates. Note that two out of $6 Y J$-dropout candidates are the objects which are also selected as $Y$-dropout candidates. The numbers of dropout candidates in the cluster field are comparable to those in the parallel field, although the cluster field is subject to the strong lensing effects. The numbers of dropout candidates are affected by two effects of the lensing magnification. One is the magnification of surface brightness that enhances the observed brightness of the dropout candidates. The other is the magnification of the observed area, which reduces the effective survey area on the source plane. In the numbers of dropout candidates, these two effects compensate (Coe et al. 2014). Albeit the small statistics of a single HFF pointing, this would be one of the reasons why the numbers of dropout candidates are similar in the cluster and the parallel fields. More quantitative arguments of the lensing effects are presented in Section 5.

We estimate photometric redshifts of our dropout candidates using the Bayesian photometric redshift code BPZ (Benítez 2000). Tables 2 3. and 44include the photometric redshifts. The redshift ranges are 5.7-7.3, 7.2-8.4, and 8.1-9.6 for the $i-, Y$, and $Y J$-dropout candidates, except a candidate with the ID of HFF1P-i12 at $z=4.5$. The three samples of dropout candidates cover $z \sim 5-10$. We confirm that the ranges of the photometric redshifts are consistent with the redshifts defined by the dropout selections, $z \sim 6-7,8$, and 9 . In the remainder of this paper, we refer to $i-, Y$-, and $Y J$-dropout candidates as $z \sim 6-7, z \sim 8, z \sim 9$ dropouts, respectively.

Recently, Atek et al. (2014b), Zheng et al. (2014), Coe et al. (2014), and Atek et al. (2014a) have identified a total of $16,18,7$, and 58 dropouts at $z>6$ 

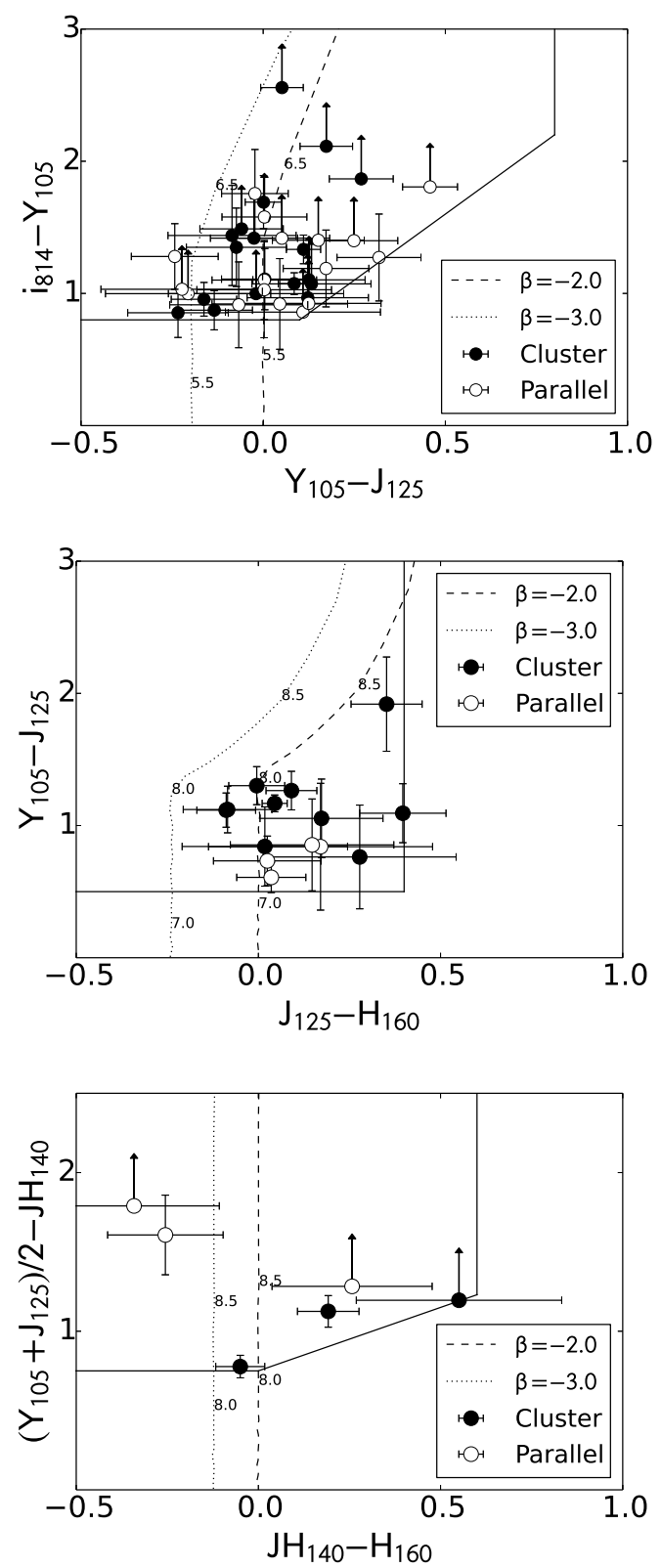

Figure 3. Two-color diagrams for $i$-dropout (top), $Y$-dropout (middle), and $Y J$-dropout candidates (bottom). The dropout selection windows are indicated with the solid lines. The filled circles (open circles) denote our dropout candidates in the cluster (parallel) field. The arrows indicate that the $i_{814}\left(Y_{105}\right.$ and $\left.J_{125}\right)$ magnitude is replaced with the $3 \sigma(1 \sigma)$ limiting magnitude for the $i$-dropout ( $Y$-dropout and $Y J$-dropout) candidates. The dashed and dotted lines present the expected colors of star-forming galaxies with UV-continuum slopes of $\beta=-2$ and -3 , respectively.

in the Abell 2744 cluster field, respectively. We recover $10,12,6$, and 25 dropouts of their samples. Laporte et al. (2014) analyze the spectral energy distribution of a $z \sim 8$ dropout, which is identified in our selection with the ID of HFF1C-Y1 and in all the other three studies. Our dropout with the ID of HFF1C-Y9 is also found in Zheng et al. (2014) as one of the three multiple images. Although we find HFF1C-Y9 by the $Y$-dropout selection, Zheng et al. (2014) identify the multiply-imaged object as an $i$-dropout. The different selections of $Y$ - and $i$ dropouts are explained by the fact that the photometric redshift of HFF1C-Y9 is 7.3 that is the border value of the $i$-dropout and $Y$-dropout redshift ranges. Zitrin et al. (2014) have reported a triply-imaged $z \sim 10$ dropout in the cluster field (see also Oesch et al. 2014). We recover one of the multiple images as a $Y J$-dropout with the ID of HFF1C-YJ1. In Section 4.4, we discuss the multiple images including HFF1C-Y9 and HFF1C-YJ1 with our mass model.

We do not recover 7,6, 1, and 33 dropouts found in Atek et al. (2014b), Zheng et al. (2014), Coe et al. (2014), and Atek et al. (2014a), respectively. The difference of our and their samples can be explained by the following three reasons. First, we use the full-depth images of HFF Abell 2744 observations, while Atek et al. (2014b), Zheng et al. (2014), and Coe et al. (2014) only use relatively shallow ACS images observed in HST Cycle 17 (GO 11689, PI: Dupke). Second, the limiting magnitude definitions are different. We take account of the space-dependent limiting magnitudes in the cluster field as described in Section 2. Third, there are differences in galaxy selection techniques. Zheng et al. (2014) and Coe et al. (2014) do not use the well-tested color selections, but sophisticated photometric redshifts for the selections. Although we concur with all 9 confident $z>8$ dropouts from Zheng et al. (2014), we recover only one $z \sim 7-8$ dropout from Zheng et al. (2014). Similarly, we recover only 19 out of 50 dropouts at $z \sim 7$ from Atek et al. (2014a). This is possibly because our selection criteria for our $z<8$ dropouts are more conservative than those of Zheng et al. (2014) and Atek et al. (2014a).

\section{MASS MODEL}

In this section, we construct a mass model of Abell 2744 at $z=0.308$ using the parametric gravitational lensing package GLAFIC (Oguri 2010). 8 Our mass model includes three types of mass distributions: cluster-scale halos, cluster member galaxy halos, and external perturbation. With the positions of multiple images provided in the literature, we optimize free parameters of the mass profiles based on a standard $\chi^{2}$ minimization to determine the best-fit mass model whose parameters are summarized in Table 5. We then calculate magnification factors $\mu$ of our dropouts and positions of the multiple images using the best-fit mass model.

\subsection{Cluster-Scale Halos}

We place three cluster-scale halos at the positions of three brightest galaxies in the core of the cluster. We adopt the Navarro-Frenk-White (NFW) profiles (Navarro et al. 1997) for the mass distributions of the cluster-scale halos. The radial profiles of NFW are described as

$$
\rho(r)=\frac{\rho_{s}}{\left(r / r_{s}\right)\left(1+r / r_{s}\right)^{2}},
$$

where $\rho_{s}$ is the characteristic density and $r_{s}$ is the scale radius. The scale radius is defined by

$$
r_{s}=\frac{r_{\mathrm{vir}}}{c_{\mathrm{vir}}}
$$

8 we name the mass model in this work 'glafic model version 1.0'. This mass model will be released on the STScI website (http://archive.stsci.edu/prepds/frontier/lensmodels/). The mass model version 2.0 is being developed, in which we include new multiple images. 


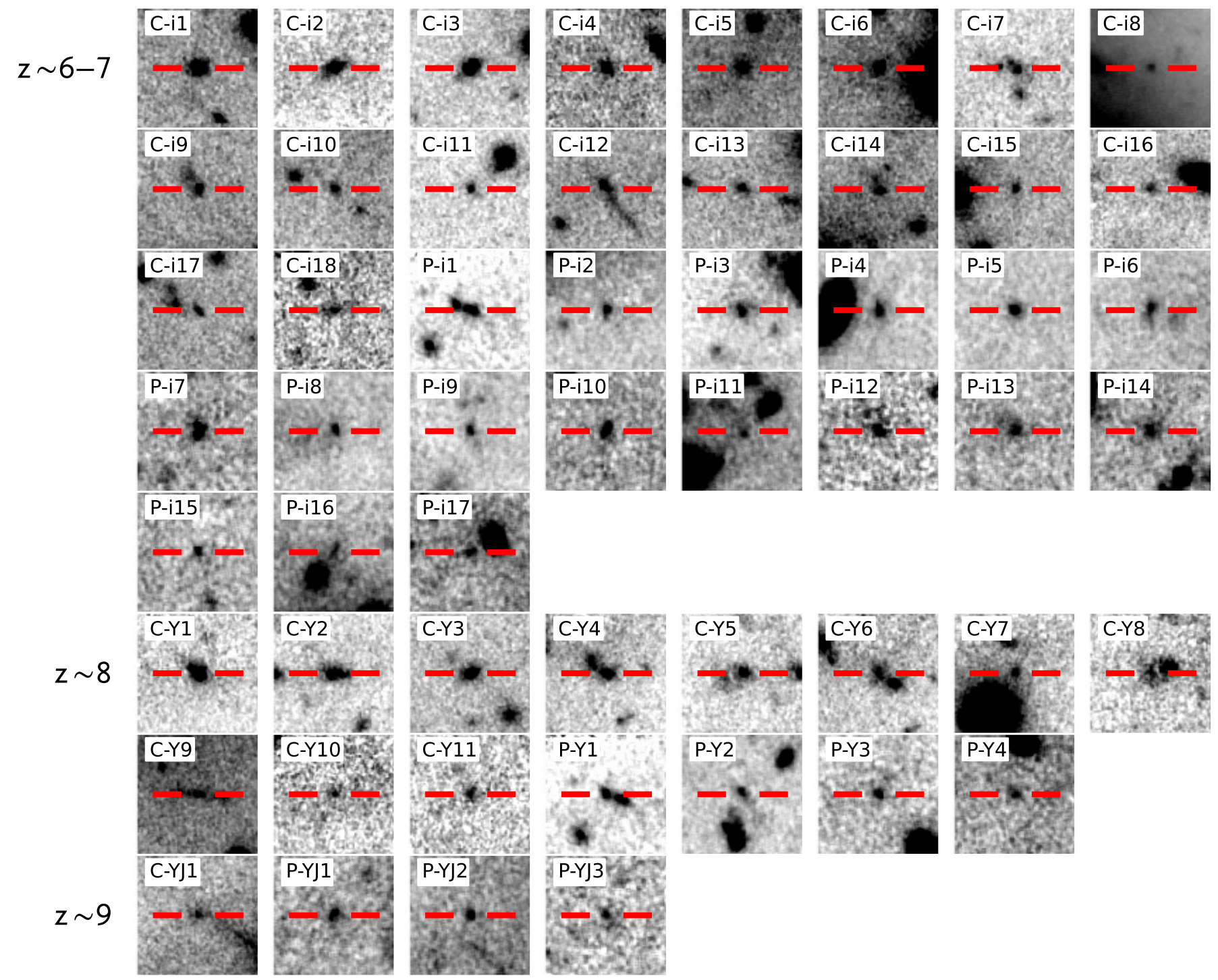

Figure 4. Cutouts of the detection images of our dropout candidates. The size of each cutout is $3^{\prime \prime} \times 3^{\prime \prime}$. Each candidate is placed at the image center, and marked with the red lines. North is up, and east is to the left.

where $r_{\text {vir }}$ is the virial radius of the cluster-scale halo and $c_{\text {vir }}$ is the concentration parameter. The scale radius and the characteristic density are related to the virial mass $M_{\text {vir }}$ and the concentration parameter with the equations,

$$
\begin{aligned}
r_{s} & =\frac{1}{c_{\mathrm{vir}}}\left(\frac{3 M_{\mathrm{vir}}}{4 \pi \Delta(z) \bar{\rho}(z)}\right)^{1 / 3}, \\
\rho_{s} & =\frac{\Delta(z) \bar{\rho}(z) c_{\mathrm{vir}}^{3}}{3 m_{\mathrm{nfw}}\left(c_{\mathrm{vir}}\right)} \\
m_{\mathrm{nfw}}\left(c_{\mathrm{vir}}\right) & =\int_{0}^{c_{\mathrm{vir}}} \frac{r}{(1+r)^{2}} d r
\end{aligned}
$$

where $\Delta(z)$ is the nonlinear overdensity (e.g., Nakamura \& Suto 1997) and $\bar{\rho}(z)$ is the mean matter density of the universe at a redshift $z$. The surface mass density $\Sigma\left(r^{\prime}\right)$ is obtained as a function of a radius $r^{\prime} \equiv \sqrt{x^{2}+y^{2}}$ by integrating $\rho(r)$ along the line of sight:

$$
\Sigma\left(r^{\prime}\right)=\int_{-\infty}^{+\infty} \rho\left(r^{\prime}, z\right) d z
$$

In the obove discussion, we assumed spherical halos. We then introduce an ellipticity $e$ in the isodensity contour by replacing $r^{\prime}$ in $\Sigma\left(r^{\prime}\right)$ (Oguri 2010):

$$
\Sigma\left(r^{\prime}\right): r^{\prime} \rightarrow \sqrt{\frac{\tilde{x}^{2}}{(1-e)}+(1-e) \tilde{y}^{2}},
$$

where $\tilde{x}$ and $\tilde{y}$ are defined by the following equations with the position angle $\theta_{e}$ (measured east of north) of the isodensity contours:

$$
\begin{array}{r}
\tilde{x}=x \cos \theta_{e}+y \sin \theta_{e} \\
\tilde{y}=-x \sin \theta_{e}+y \cos \theta_{e} .
\end{array}
$$

We use the ellipsoidal halos in this work. Each clusterscale halo has four free parameters: $M_{\mathrm{vir}}, c_{\mathrm{vir}}, e$, and $\theta_{e}$. 
Table 2

Dropout candidates at $z \sim 6-7$ in the HFF Abell 2744 Fields

\begin{tabular}{|c|c|c|c|c|c|c|c|c|}
\hline ID & R.A. (J2000) & Dec (J2000) & $i_{814}-Y_{105}$ & $Y_{105}-J_{125}$ & $J_{125^{\mathrm{a}}}$ & Magnification $^{\mathrm{b}}$ & Photo- $z$ & Reference $^{\mathrm{c}}$ \\
\hline \multicolumn{9}{|l|}{ Cluster field } \\
\hline HFF1C-i1 & 3.593804 & -30.415447 & $>2.37$ & $0.05 \pm 0.07$ & $26.10 \pm 0.05$ & $3.73^{+0.24}$ & $6.6 \pm 0.8$ & 1,3 \\
\hline HFF1C-i2 & 3.570654 & -30.414659 & $1.33 \pm 0.12$ & $0.11 \pm 0.05$ & $26.21 \pm 0.03$ & $1.62 \pm 0.06$ & $6.0 \pm 0.7$ & 1,3 \\
\hline HFF1C-i3 & 3.606222 & -30.386644 & $1.07 \pm 0.09$ & $0.09 \pm 0.04$ & $26.25 \pm 0.04$ & $1.69 \pm 0.05$ & $5.8 \pm 0.7$ & 1,3 \\
\hline $\mathrm{HFF} 1 \mathrm{C}-\mathrm{i} 4$ & 3.606385 & -30.407282 & $1.69 \pm 0.21$ & $0.00 \pm 0.05$ & $26.37 \pm 0.04$ & $2.25_{-0.10}^{+0.12}$ & $6.3 \pm 0.7$ & 1,3 \\
\hline HFF1C-i5 & 3.580452 & -30.405043 & $>2.10$ & $0.17 \pm 0.07$ & $26.60 \pm 0.05$ & $5.64 \pm 0.39$ & $6.8 \pm 0.8$ & 1,3 \\
\hline HFF1C-i6 & 3.597834 & -30.395961 & $>1.71$ & $0.27 \pm 0.09$ & $26.79 \pm 0.07$ & $2.87 \pm 0.19$ & $7.0 \pm 0.8$ & 1,3 \\
\hline HFF1C-i7 & 3.590761 & -30.379408 & $0.95 \pm 0.13$ & $-0.16 \pm 0.09$ & $27.06 \pm 0.08$ & $1.87_{-0.05}^{+0.06}$ & $5.9 \pm 0.7$ & $\ldots$ \\
\hline $\mathrm{HFF} 1 \mathrm{C}-\mathrm{i} 8$ & 3.585321 & -30.397958 & $>1.10$ & $0.13 \pm 0.15$ & $27.19 \pm 0.10$ & $4.60_{-0.48}^{+0.43}$ & $6.8 \pm 0.8$ & 1,3 \\
\hline HFF1C-i9 & 3.601072 & -30.403991 & $1.11 \pm 0.27$ & $0.00 \pm 0.11$ & $27.26 \pm 0.09$ & $3.56_{-0.23}^{+0.26}$ & $5.9 \pm 0.7$ & 1,3 \\
\hline $\mathrm{HFF} 1 \mathrm{C}-\mathrm{i} 10$ & 3.600619 & -30.410296 & $>1.43$ & $-0.06 \pm 0.11$ & $27.29 \pm 0.08$ & $11.43_{-1.20}^{+1.60}$ & $6.4 \pm 0.7$ & 3 \\
\hline HFF $1 \mathrm{C}-\mathrm{i} 11$ & 3.603426 & -30.383219 & $0.87 \pm 0.16$ & $-0.13 \pm 0.11$ & $27.29 \pm 0.09$ & $1.71_{-0.05}^{+0.04}$ & $5.8 \pm 0.7$ & 3 \\
\hline HFF1C-i12 & 3.603214 & -30.410350 & $>1.36$ & $-0.03 \pm 0.12$ & $27.32 \pm 0.09$ & $3.88_{-0.21}^{+0.29}$ & $6.3 \pm 0.7$ & $1,2,3$ \\
\hline HFF $1 \mathrm{C}-\mathrm{i} 13$ & 3.592944 & -30.413328 & $>1.25$ & $-0.09 \pm 0.20$ & $27.35 \pm 0.15$ & $6.85_{-0.54}^{+0.60}$ & $6.1 \pm 0.7$ & 3 \\
\hline HFF1C-i14 & 3.585016 & -30.413084 & $0.85 \pm 0.19$ & $-0.23 \pm 0.14$ & $27.45 \pm 0.12$ & $2.94_{-0.17}^{+0.18}$ & $5.7_{-1.1}^{+0.7}$ & $\ldots$ \\
\hline HFF1C-i15 & 3.576889 & -30.386329 & $>0.96$ & $0.13 \pm 0.18$ & $27.45 \pm 0.16$ & $2.77_{-0.13}^{+0.15}$ & $6.1_{-0.7}^{+0.8}$ & 3 \\
\hline HFF1C-i16 & 3.609003 & -30.385283 & $1.35 \pm 0.33$ & $-0.07 \pm 0.14$ & $27.56 \pm 0.12$ & $1.59 \pm 0.04$ & $6.1 \pm 0.7$ & 3 \\
\hline HFF1C-i17 & 3.604563 & -30.409364 & $>0.91$ & $0.12 \pm 0.16$ & $27.62 \pm 0.11$ & $2.94_{-0.16}^{+0.19}$ & $6.1 \pm 0.8$ & 3 \\
\hline HFF1C-i18 & 3.590518 & -30.379763 & $>0.95$ & $-0.02 \pm 0.25$ & $28.09 \pm 0.19$ & $\begin{array}{l}1.94_{-0.05}^{+0.06} \\
+0.06\end{array}$ & $6.1_{-1.4}^{+0.9}$ & 3 \\
\hline \multicolumn{9}{|l|}{ Parallel field } \\
\hline HFF1P-i1 & 3.474802 & -30.362578 & $>1.80$ & $0.46 \pm 0.07$ & $26.52 \pm 0.05$ & 1.04 & $7.3 \pm 0.8$ & $\ldots$ \\
\hline HFF1P-i2 & 3.480642 & -30.371175 & $1.76 \pm 0.34$ & $-0.02 \pm 0.09$ & $26.95 \pm 0.07$ & 1.05 & $6.3 \pm 0.7$ & $\ldots$ \\
\hline HFF1P-i3 & 3.487575 & -30.364380 & $1.27 \pm 0.33$ & $0.32 \pm 0.11$ & $27.06 \pm 0.08$ & 1.05 & $5.8 \pm 0.7$ & $\ldots$ \\
\hline HFF1P-i4 & 3.488924 & -30.394630 & $>1.39$ & $0.25 \pm 0.11$ & $27.14 \pm 0.08$ & 1.05 & $6.7 \pm 0.8$ & $\ldots$ \\
\hline HFF1P-i5 & 3.482550 & -30.371559 & $1.19 \pm 0.29$ & $0.17 \pm 0.11$ & $27.15 \pm 0.09$ & 1.05 & $5.8_{-1.4}^{+0.7}$ & $\ldots$ \\
\hline HFF1P-i6 & 3.483960 & -30.397152 & $>1.57$ & $0.00 \pm 0.11$ & $27.20 \pm 0.09$ & 1.05 & $6.3 \pm 0.7$ & $\ldots$ \\
\hline HFF1P-i7 & 3.467582 & -30.396908 & $>1.39$ & $0.15 \pm 0.12$ & $27.23 \pm 0.09$ & 1.04 & $6.8 \pm 0.8$ & $\ldots$ \\
\hline HFF1P-i8 & 3.467097 & -30.387686 & $1.28 \pm 0.25$ & $-0.24 \pm 0.11$ & $27.30 \pm 0.10$ & 1.04 & $6.0 \pm 0.7$ & $\ldots$ \\
\hline HFF1P-i9 & 3.489520 & -30.399528 & $>1.41$ & $0.05 \pm 0.13$ & $27.32 \pm 0.10$ & 1.05 & $6.6 \pm 0.8$ & $\ldots$ \\
\hline HFF1P-i10 & 3.466056 & -30.394409 & $1.10 \pm 0.30$ & $0.00 \pm 0.14$ & $27.43 \pm 0.11$ & 1.04 & $6.0 \pm 0.7$ & $\ldots$ \\
\hline HFF1P-i11 & 3.460587 & -30.366320 & $0.92 \pm 0.34$ & $0.05 \pm 0.18$ & $27.70 \pm 0.14$ & 1.04 & $5.8_{-1.1}^{+0.7}$ & $\ldots$ \\
\hline HFF1P-i12 & 3.455844 & -30.366359 & $1.03 \pm 0.36$ & $0.00 \pm 0.18$ & $27.70 \pm 0.14$ & 1.03 & $4.5_{-3.9}^{+1.6}$ & $\ldots$ \\
\hline HFF1P-i13 & 3.488139 & -30.367864 & $>0.91$ & $0.12 \pm 0.19$ & $27.73 \pm 0.15$ & 1.06 & $\begin{array}{r}-3.9 \\
5.8_{-5.2}^{+1.0}\end{array}$ & $\ldots$ \\
\hline HFF1P-i14 & 3.486988 & -30.399579 & $0.91 \pm 0.33$ & $-0.07 \pm 0.18$ & $27.75 \pm 0.15$ & 1.05 & $5.9_{-1 .}^{+0.2}$ & $\ldots$ \\
\hline HFF1P-i15 & 3.484920 & -30.376917 & $>0.85$ & $0.11 \pm 0.20$ & $27.82 \pm 0.16$ & 1.05 & $5.9_{-0.7}^{+-1.1}$ & $\ldots$ \\
\hline HFF1P-i16 & 3.477238 & -30.385998 & $>1.02$ & $-0.22 \pm 0.21$ & $27.98 \pm 0.18$ & 1.05 & $6.5 \pm 0.7$ & $\ldots$ \\
\hline HFF1.P-i17 & 3.481928 & -30.389557 & $>0.99$ & $-0.21 \pm 0.22$ & $27.99 \pm 0.19$ & 1.05 & $6.2_{-0.8}^{+0.7}$ & $\ldots$ \\
\hline
\end{tabular}

a Total magnitudes estimated with the aperture correction.

b The magnification errors in the parallel field are less than $1 \%$ based on our model extrapolation estimates. Note that the errors in the parallel field would be underestimated because Abell 2744 is a complex merging cluster.

c References: (1) Atek et al. (2014b); (2) Zheng et al. (2014); (3) Atek et al. (2014a)

\subsection{Cluster Member Galaxy Halos}

To estimate contributions from cluster member galaxy halos, we identify cluster member galaxies with spectroscopic redshifts $z_{\mathrm{spec}}$, photometric redshifts $z_{\text {photo }}$, and $B_{435}-V_{606}$ colors. First, we use $z_{\text {spec }}$ presented in Table 5 of Owers et al. (2011). Galaxies at $0.28<z_{\text {spec }}<0.34$ are regarded as cluster member galaxies. For objects with no $z_{\text {spec }}$, we apply color criteria,

$$
\begin{aligned}
-\frac{1}{18}\left(B_{435}-V_{606}\right)+2 & <V_{606}, \\
-\frac{1}{18}\left(B_{435}-V_{606}\right)+2.4 & >V_{606}, \\
V_{606} & <24,
\end{aligned}
$$

and select galaxies on the red-sequence of the cluster redshift. Figure 5 presents the $B_{435}-V_{606}$ versus $V_{606}$ color-magnitude diagram of our objects, together with the color criteria of the red-sequence galaxies shown with the solid-line box. Finally, for objects that are selected neither by $z_{\text {spec }}$ nor the red-sequence criteria, we refer $z_{\text {photo }}$ estimated with BPZ (Section 3). We select member galaxies with the $z_{\text {photo }}$ criterion and the relaxed colormagnitude criteria,

$$
\begin{aligned}
0.09<z_{\text {photo }} & <0.4, \\
-\frac{1}{22}\left(B_{435}-V_{606}\right)+1.2 & <V_{606}, \\
-\frac{1}{22}\left(B_{435}-V_{606}\right)+3 & >V_{606}, \\
V_{606} & <26 .
\end{aligned}
$$

The dashed-line box in Figure 5 indicates the boundary of the relaxed color-magnitude criteria.

We describe halo mass distributions of these member galaxies by the sum of pseudo-Jaffe ellipsoids (PJE; Keeton 2001, see also Jaffe 1983). In this model, the mass profile is characterized by the velocity dispersion $\sigma$ and 
Table 3

Dropout candidates at $z \sim 8$ in the HFF Abell 2744 Fields

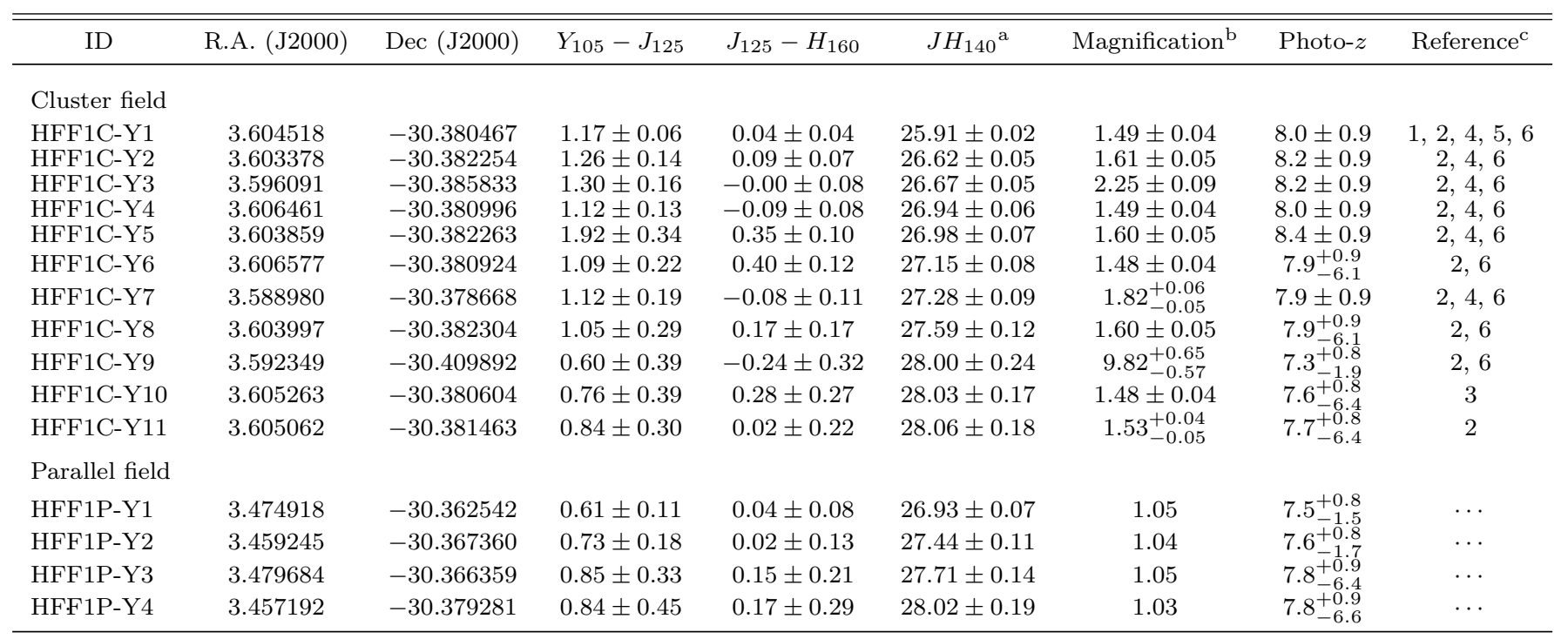

a Total magnitudes estimated with the aperture correction.

b The magnification errors in the parallel field are less than $1 \%$

c References: (1) Atek et al. (2014b); (2) Zheng et al. (2014); (3) Zheng et al. (2014) possible candidates; (4) Coe et al. (2014); (5) Laporte et al. (2014); (6) Atek et al. (2014a).

Table 4

Dropout candidates at $z \sim 9$ in the HFF Abell 2744 Fields

\begin{tabular}{|c|c|c|c|c|c|c|c|c|}
\hline ID & R.A. (J2000) & Dec (J2000) & $\left(Y_{105}+J_{125}\right) / 2-J H_{140}$ & $J H_{140}-H_{160}$ & $H_{160}{ }^{\mathrm{a}}$ & Magnification $^{\mathrm{b}}$ & Photo- $z$ & Reference $^{\mathrm{c}}$ \\
\hline \multicolumn{9}{|l|}{ Cluster field } \\
\hline HFF1C-YJ1 & 3.592512 & -30.401486 & $>1.22$ & $0.55 \pm 0.30$ & $27.37 \pm 0.16$ & $14.40_{-1.06}^{+1.20}$ & $9.6_{-7.1}^{+1.0}$ & 1,2 \\
\hline $\mathrm{HFF} 1 \mathrm{C}-\mathrm{Y} 2^{\mathrm{d}}$ & 3.603380 & -30.382255 & $0.78 \pm 0.07$ & $-0.05 \pm 0.07$ & $26.67 \pm 0.05$ & $1.61 \pm 0.05$ & $8.2 \pm 0.9$ & $3,4,5$ \\
\hline HFF1C-Y $5^{d}$ & 3.603859 & -30.382262 & $1.13 \pm 0.10$ & $0.19 \pm 0.08$ & $26.78 \pm 0.05$ & $1.60 \pm 0.05$ & $8.4 \pm 0.9$ & $3,4,5$ \\
\hline \multicolumn{9}{|l|}{ Parallel field } \\
\hline HFF1P-YJ1 & 3.488893 & -30.396183 & $1.61 \pm 0.25$ & $-0.26 \pm 0.16$ & $27.67 \pm 0.11$ & 1.05 & $8.7 \pm 1.0$ & $\ldots$ \\
\hline HFF1P-YJ2 & 3.473522 & -30.384024 & $>1.28$ & $0.26 \pm 0.22$ & $27.70 \pm 0.11$ & 1.04 & $8.8_{-1.7}^{+1.0}$ & $\ldots$ \\
\hline HFF1P-YJ3 & 3.474445 & -30.368728 & $>1.79$ & $-0.34 \pm 0.23$ & $28.14 \pm 0.17$ & 1.04 & $8.9_{-6.9}^{+1.0}$ & $\cdots$ \\
\hline
\end{tabular}

${ }^{a}$ Total magnitudes estimated with the aperture correction.

b The magnification errors in the parallel field are less than $1 \%$

c References: (1) Zitrin et al. (2014); (2) Oesch et al. (2014); (3) Zheng et al. (2014); (4) Coe et al. (2014); (5) Atek et al. (2014a).

d Identified by our two selections for $Y$-dropouts and $Y J$-dropouts.

Table 5

Best-fit mass model parameters

\begin{tabular}{|c|c|c|c|c|c|c|c|}
\hline Component & model & Mass $\left(h^{-1} M_{\odot}\right)$ & $e$ & $\theta_{e}\left(^{\circ}\right)$ & $c$ & R.A. (J2000) & Dec (J2000) \\
\hline Cluster halo 1 & NFW & $3.5 \times 10^{14}$ & 0.20 & 30.0 & 3.41 & 3.585972 & -30.400122 \\
\hline Cluster halo 2 & NFW & $2.5 \times 10^{14}$ & 0.49 & -41.9 & 8.01 & 3.592074 & -30.405165 \\
\hline \multirow[t]{2}{*}{ Cluster halo 3} & NFW & $1.3 \times 10^{13}$ & 0.60 & 72.2 & 28.2 & 3.583417 & -30.392069 \\
\hline & & $\sigma_{*}\left(\mathrm{~km} \mathrm{~s}^{-1}\right)$ & $r_{\text {trun }, *}\left({ }^{\prime \prime}\right)$ & $\eta$ & & & \\
\hline \multirow[t]{2}{*}{ Member galaxies } & PJE & $2.0 \times 10^{2}$ & $5.09 \times 10$ & 1.22 & & & \\
\hline & & $z_{s, \mathrm{fid}}$ & $\gamma$ & $\theta_{\gamma}\left({ }^{\circ}\right)$ & $\kappa$ & & \\
\hline Perturbation & PRT & $2.0($ fix $)$ & $6.21 \times 10^{-2}$ & 15.2 & 0.0 (fix) & & \\
\hline
\end{tabular}




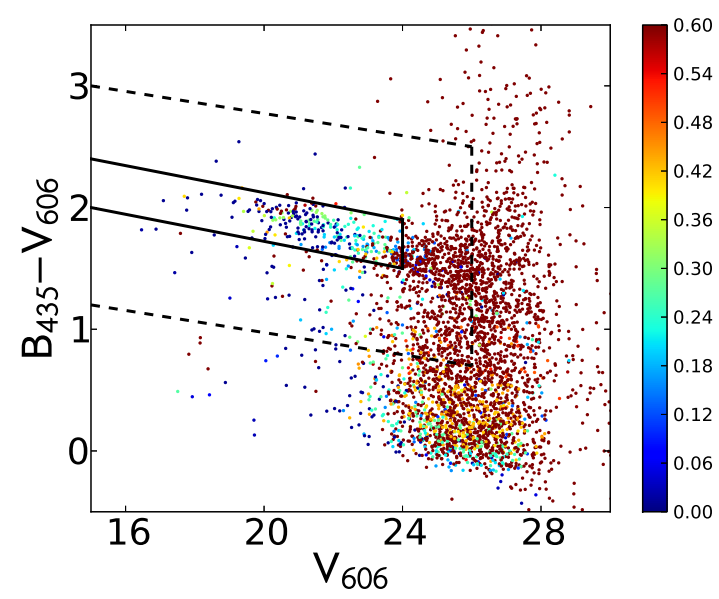

Figure 5. Color-magnitude diagram for the objects (circles) detected in the optical images. The color code for the circles indicates photometric redshifts estimated with BPZ. The solid line box indicates the color selection window for the cluster members of the red-sequence galaxies at the cluster redshift. The dashed line box represents the relaxed selection window that is applied with the photometric redshift criterion.

the truncation radius $r_{\text {trun }}$. We assume that the parameters of $\sigma$ and $r_{\text {trun }}$ are scaled with the galaxy luminosity $L$ in the $i_{814}$ band,

$$
\begin{aligned}
\frac{\sigma}{\sigma_{*}} & =\left(\frac{L}{L_{*}}\right)^{1 / 4}, \\
\frac{r_{\text {trun }}}{r_{\text {trun }, *}} & =\left(\frac{L}{L_{*}}\right)^{\eta},
\end{aligned}
$$

where $L_{*}$ is the normalization luminosity at the cluster redshift, and $\sigma_{*}, r_{\text {trun,* }}$, and $\eta$ are free parameters. The mass-to-light ratio is constant for $\eta=0.5$. The ellipticities and position angles of the member galaxy halos are determined from shapes of the member galaxies in the $J H_{140}$ band.

\subsection{External Perturbation}

Although the mass distribution of Abell 2744 is mostly explained by the contributions from the three clusterscale halos (Section 4.1) and the member galaxy halos (Section 4.2), we include perturbation induced by external sources to improve our mass model. If the perturbation is weak, its potential can be described by (e.g., Kochanek 1991)

$$
\phi=\frac{1}{2} r^{2} \kappa+\frac{1}{2} r^{2} \gamma \cos 2\left(\theta-\theta_{\gamma}\right)
$$

where $\kappa$ is the constant convergence and $\gamma$ is the constant tidal shear. The amplitude of the potential $\phi$ is defined for a given fiducial source redshift $z_{\mathrm{s} \text {,fid }}$. We refer to this potential as PRT. In this paper, $\gamma$ and $\theta_{\gamma}$ are free parameters. We fix $z_{s \text {,fid }}$ and $\kappa ; z_{s, \text { fid }} \equiv 2.0$ and $\kappa \equiv 0$.

\subsection{Model Optimization}

To constrain the mass-model parameters of the cluster, we use the positions of multiply imaged systems. We identify multiply imaged galaxies based on their colors and morphologies while iteratively refining the massmodel parameters. In total, we use the positions of 67 multiple images of 24 systems summarized in Table 6 Seventeen out of the 24 systems, IDs $1.1-17.2$ (Table 6), are the same as those listed in Table 1 of the document provided by the HFF map-making team (PI: K. Sharon; see also Johnson et al. 2014) 9 Similarly, four highredshift systems from the 24 systems, IDs $19.1-22.2$, are listed in Table 3 of Atek et al. (2014b), among which IDs $19.1,19.2$, and 19.3 are identified as $z \sim 6-7$ dropouts in Section 3.2 The images of IDs 19.1, 19.2, and 19.3 are referred to as HFF1C-i5, HFF1C-i6, and HFF1C-i8 in Table 2. We also find three new sets of multiple images, IDs $18.1-18.3$ and $23.1-24.2$.

We search for the best-fit mass model which reproduces the positions of the multiple images. We optimize the 23 free parameters described in Section 4.1 4.3 based on a $\chi^{2}$ minimization with the downhill-simplex algorithm (See Oguri 2010 for more details). We assign a positional error of 0.44 in the image plane for each multiple image, following Oguri (2010), and obtain the best-fit parameters as summarized in Table [5. The best-fit model has $\chi^{2}=52.8$ for 41 degrees of freedom, suggesting that our model is reasonable.

In the following sections, we use this best-fit mass model to estimate the lensing effects both in the cluster and parallel fields. Because the parallel field has no multiple images to constrain the parameters of the mass model, it should be noted that the lensing effects in the parallel field are estimated from the extrapolation of the mass model determined at the cluster field. However, the extrapolation gives negligibly small uncertainties in our final results, due to the very small magnification factors (see Section 4.5).

\subsection{Magnification Factors and Multiple Images}

Figure 6 displays the critical lines for $z=8$ sources and the positions of our $z \sim 5-10$ dropouts in the cluster field. Because most of the dropouts are located far from the critical lines, the magnification factors are generally small, $\mu \sim 1.5-2$, as presented in Tables 2, 4. However, some of the dropouts are placed near the critical line and highly magnified. In particular, the magnification factors of three dropouts, HFF1C-i10, HFF1C-Y9, and HFF1C-YJ1, are estimated to be $\mu \sim 10-14$. The intrinsic absolute magnitudes of these three dropouts are $-17.00,-16.66$, and $-17.06 \mathrm{mag}$, respectively. Figure 7 shows the positions of our $z \sim 5-10$ dropouts in the parallel field. The magnification factors in the parallel field are almost the same and near unity, typically $\sim 1.05$. Thus, the lensing effects in the parallel field are negligibly small, and the parallel field may be regarded as a blank field. Although the magnification of the parallel field is very small, we adopt the lensing magnifications to our dropouts both in the cluster and the parallel fields in our analysis. We estimate the errors of the magnification factors with a Markov Chain Monte Carlo (MCMC) method. These errors are shown in Tables 2,4.

Our mass model predicts that three systems in our dropout samples have counter images. We discuss the positions and the redshifts of these multiple images using our mass model. Because the predicted positions of multiple images depend on the source redshifts, we esti-

\footnotetext{
9 http://archive.stsci.edu/prepds/frontier/lensmodels/ hlsp_frontier_model_abell2744_sharon_v1_readme.pdf
} 
Table 6

Multiple images

\begin{tabular}{|c|c|c|c|c|c|c|}
\hline ID & R.A. (J2000) & $\operatorname{Dec}(\mathrm{J} 2000)$ & $z_{\text {model }}{ }^{\mathrm{a}}$ & $z_{\text {photo }}{ }^{b}$ & $z_{\text {spec }}{ }^{b}$ & Reference $^{\mathrm{c}}$ \\
\hline 1.1 & 3.595958 & -30.40682 & \multirow[t]{3}{*}{1.61} & \multirow[t]{3}{*}{$0.5-2.2$} & \multirow[t]{3}{*}{$\cdots$} & \multirow[t]{3}{*}{1} \\
\hline 1.2 & 3.597542 & -30.40392 & & & & \\
\hline 1.3 & 3.586208 & -30.40999 & & & & \\
\hline 2.1 & 3.596417 & -30.40612 & \multirow[t]{3}{*}{1.63} & \multirow[t]{3}{*}{$0.5-2.2$} & \multirow[t]{3}{*}{$\cdots$} & \multirow[t]{3}{*}{1} \\
\hline 2.2 & 3.597042 & -30.40475 & & & & \\
\hline 2.3 & 3.585744 & -30.41010 & & & & \\
\hline 3.1 & 3.585417 & -30.39990 & \multirow[t]{4}{*}{2.00} & \multirow[t]{4}{*}{$0.5-2.9$} & \multirow[t]{4}{*}{$\cdots$} & \multirow[t]{4}{*}{1} \\
\hline 3.2 & 3.583250 & -30.40335 & & & & \\
\hline 3.3 & 3.597292 & -30.39672 & & & & \\
\hline 3.4 & 3.586417 & -30.40213 & & & & \\
\hline 4.1 & 3.596750 & -30.39630 & 1.97 & $0.5-2.9$ & $\cdots$ & 1 \\
\hline 4.2 & 3.582542 & -30.40227 & & & & \\
\hline 4.3 & 3.586250 & -30.40085 & & & & \\
\hline 4.4 & 3.584500 & -30.39929 & & & & \\
\hline 5.1 & 3.589375 & -30.39388 & 2.17 & $\cdots$ & 3.98 & 1 \\
\hline 5.2 & 3.588792 & -30.39380 & & & & \\
\hline 5.3 & 3.577500 & -30.39957 & & & & \\
\hline 6.1 & 3.592125 & -30.40263 & $3.58^{\mathrm{d}}$ & $\ldots$ & 3.58 & 1 \\
\hline 6.2 & 3.595625 & -30.40162 & & & & \\
\hline 6.3 & 3.580417 & -30.40892 & & & & \\
\hline 6.4 & 3.593208 & -30.40491 & & & & \\
\hline 6.5 & 3.593583 & -30.40511 & & & & \\
\hline 7.1 & 3.583417 & -30.39207 & 1.18 & $\cdots$ & & 1 \\
\hline 7.2 & 3.585000 & -30.39138 & & & & \\
\hline 7.3 & 3.579958 & -30.39476 & & & & \\
\hline 8.1 & 3.598535 & -30.40180 & $2.02^{\mathrm{d}}$ & $\ldots$ & 2.019 & 1 \\
\hline 8.2 & 3.594042 & -30.40801 & & & & \\
\hline 8.3 & 3.586417 & -30.40937 & & & & \\
\hline 9.1 & 3.598261 & -30.40232 & 2.71 & $0.3-3.4$ & $\cdots$ & 1 \\
\hline 9.2 & 3.595233 & -30.40741 & & & & \\
\hline 9.3 & 3.584601 & -30.40982 & & & & \\
\hline 10.1 & 3.589708 & -30.39434 & 2.59 & $\cdots$ & $\cdots$ & 1 \\
\hline 10.2 & 3.588833 & -30.39422 & & & & \\
\hline 11.1 & 3.588375 & -30.40527 & 3.76 & $0.6-2.8$ & $\cdots$ & 1 \\
\hline 11.2 & 3.587125 & -30.40624 & & & & \\
\hline 11.3 & 3.600150 & -30.39715 & & & & \\
\hline 12.1 & 3.588417 & -30.40588 & 5.38 & $1.8-3.2$ & $\cdots$ & 1 \\
\hline 12.2 & 3.587375 & -30.40648 & & & & \\
\hline 12.3 & 3.600721 & -30.39709 & & & & \\
\hline 13.1 & 3.597264 & -30.40143 & 2.73 & $0.4-2.8$ & $\cdots$ & 1 \\
\hline 13.2 & 3.582792 & -30.40891 & & & & \\
\hline 14.1 & 3.593239 & -30.40325 & 3.32 & $1.4-3.1$ & $\cdots$ & 1 \\
\hline 14.2 & 3.594555 & -30.40300 & & & & \\
\hline 15.1 & 3.592375 & -30.40256 & 1.46 & $0.6-2.6$ & $\cdots$ & 1 \\
\hline 15.2 & 3.593792 & -30.40216 & & & & \\
\hline 15.3 & 3.582792 & -30.40804 & & & & \\
\hline 16.1 & 3.589750 & -30.39464 & 1.79 & $1.8-3.2$ & $\cdots$ & 1 \\
\hline 16.2 & 3.588458 & -30.39444 & & & & \\
\hline 17.1 & 3.590750 & -30.39556 & 1.44 & $1.5-5.4$ & $\cdots$ & 1 \\
\hline 17.2 & 3.588375 & -30.39564 & & & & \\
\hline 18.1 & 3.598676 & -30.40491 & 2.19 & $\cdots$ & $\cdots$ & $\cdots$ \\
\hline 18.2 & 3.587053 & -30.41126 & & & & \\
\hline 18.3 & 3.596818 & -30.40783 & & & & \\
\hline 19.1 & 3.580452 & -30.40504 & 7.94 & $\cdots$ & $\cdots$ & 2 \\
\hline 19.2 & 3.597831 & -30.39596 & & & & \\
\hline 19.3 & 3.585321 & -30.39796 & & & & \\
\hline 20.1 & 3.596572 & -30.40900 & 7.50 & $\cdots$ & $\cdots$ & 2 \\
\hline 20.2 & 3.600058 & -30.40440 & & & & \\
\hline 20.3 & 3.585801 & -30.41175 & & & & \\
\hline 21.1 & 3.591432 & -30.39669 & 4.64 & $\cdots$ & $\cdots$ & 2 \\
\hline 21.2 & 3.576122 & -30.40449 & & & & \\
\hline 22.1 & 3.593552 & -30.40971 & 3.73 & $\cdots$ & $\cdots$ & 2 \\
\hline 22.2 & 3.600541 & -30.40182 & & & & \\
\hline 23.1 & 3.578090 & -30.39964 & 1.75 & $\cdots$ & $\cdots$ & $\cdots$ \\
\hline 23.2 & 3.589237 & -30.39444 & & & & \\
\hline 24.1 & 3.584284 & -30.40893 & 2.62 & $\cdots$ & $\cdots$ & \\
\hline 24.2 & 3.598125 & -30.40098 & & & & \\
\hline
\end{tabular}

a Redshift from the best-fit mass model.

b Numbers quoted from Johnson et al. (2014).

c References: (1) http://archive.stsci.edu/prepds/frontier/lensmodels/hlsp_frontier_model_abell2744_sharon_v1_readme.pdf see also Johnson et al. (2014); (2) Atek et al. (2014b)

d Fixed to $z_{\text {spec }}$. 
mate the redshifts of multiple images from the positions in the image plane.

HFF1C-YJ1 at $z \sim 9-$ HFF1C-YJ1 is a highly magnified dropout at $z \sim 9$ which is also reported in Zitrin et al. (2014). Zitrin et al. (2014) claim two counter images of this dropout, which we refer to as HFF1C-YJ1-2 and HFF1C-YJ1-3. In Figure 8, we show the predicted positions of the counter images of HFF1C-YJ1 whose redshifts are assumed to be $z=4-12$. The positions of the counter images are consistent with our estimates, if the redshift of HFF1C-YJ1 is $z>6$. In fact, the predicted positions of the counter images are largely separated, $>2^{\prime \prime}$, if HFF1C-YJ1 resides at $z=4$. Thus our mass model predicts that the redshift of HFF1CYJ1 is $z>6$, which is consistent with the findings of Zitrin et al. (2014). The multiple image positions rule out the possibility that HFF1C-YJ1 is a low redshift galaxy at $z<4$, and imply that the HFF1C-YJ1 system is a strong candidate of a high redshift galaxy. Combining the result of photometric redshift, we find that the system of HFF1C-YJ1, HFF1C-YJ1-2 and HFF1C-YJ1-3 is located at $z \simeq 9.6$.

HFF1C-Y9 at $z \sim 8-$ HFF1C-Y9 is a highly magnified dropout at $z \sim 8$. Zheng et al. (2014) find two counter images of this dropout, which are referred to as HFF1C-Y9-2 and HFF1C-Y9-3 in this paper. Figure 9 presents the predicted positions of the counter images of HFF1C-Y9. Our mass model predicts that HFF1CY9 has more than two counter images, if HFF1C-Y9 resides at $z=6-8$. However, we cannot investigate the positions of these additional counter images due to the bright galaxies along the lines of sight. We compare the observed images of HFF1C-Y9-2 and HFF1C-Y9-3 with the predicted positions. The observed images fall in the predicted positions for the system at $z=4-6$, implying that HFF1C-Y9 would be a source at a redshift slightly lower than that estimated in the dropout selection.

HFF1C-i5, -i6, and -is at $z \sim 6-7$ - We find three multiple images of an $i$-dropout, which are HFF1C-i5, HFF1C$\mathrm{i} 6$, and $\mathrm{HFF} 1 \mathrm{C}-\mathrm{i} 8$. We use the positions of these three multiple images for the construction of our mass model. Their IDs in Table 6 are 19.1, 19.2, and 19.3, respectively. In addition to these three multiple images, Atek et al. (2014b) report another counter images, which is named Image 5.4 in Table 3 of Atek et al. (2014b). In our paper, we refer to Image 5.4 as HFF1C-i5-2. In Figure 10. we plot the predicted positions of these four multiple images. The predicted position of HFF1C-i5-2 is about $8^{\prime \prime}$ away from the images reported by Atek et al. (2014b) . Instead, it is close to the position predicted by Jauzac et al. (2014) shown with the green circle in Figure 10. The observed images of the other three multiple images lie near the predicted positions at $z=6$ and 8 . Our mass model predicts that the best-fit value of their redshift is $z=7.94$, as shown in Table 6 .

\subsection{Comparisons with the Public Mass Models}

Mass models of Abell 2744 are also made by other groups (e.g., Lam et al. 2014). Eight public mass models are accessible through the STScI website ${ }^{10}$ which are

10 http://archive.stsci.edu/prepds/frontier/lensmodels/ made by the five independent groups, M. Bradač (PI), The Clusters As TelescopeS (CATS) team (Co-PI's J.P Kneib, P. Natarajan; see Richard et al. 2014), J. Merten \& A. Zitrin (Co-PI's), K. Sharon (PI; see Johnson et al. 2014), and L. Williams (PI). Figure 11 compares the magnification factors of our mass model and these public mass models at the positions of our dropout candidates in the cluster field. The vertical axes show $\Delta \mu / \mu$, where $\mu$ is the magnification factor from our mass model and $\Delta \mu \equiv \mu_{\text {other }}-\mu$ is the difference between magnification factors of our model and a public mass model $\left(\mu_{\text {other }}\right)$. In the cluster field, the magnification factors from our mass model are broadly consistent with those from the public mass models. We especially find excellent agreements with the CATS and Zitrin-NFW models. The Merten's group extends their mass model to the parallel field using weak lensing data covering both the cluster and parallel field. The magnification factors in the parallel field from the Merten model are $\sim 1.08-1.22$. Our mass model estimates the magnifications of the dropouts in the parallel field to be $\sim 1.05$, which is consistent with those from Merten model.

\section{UV LUMINOSITY FUNCTIONS}

In this section, we derive UV luminosity functions of dropout galaxies at $z \sim 6-7,8$, and 9 based on the $z \sim 5-10$ dropouts identified by our HFF study and the previous blank field surveys. In Section 5.1, we estimate the contamination rates of our dropout samples. In Section 5.2 we conduct Monte Carlo simulations with the gravitational lensing effects, and in Section 5.3 we obtain simulated number counts of dropouts in the image plane. Incorporating the contamination estimates, we search for the best-fit Schechter parameters for the UV luminosity functions of dropout galaxies at $z \sim 6-7,8$, and 9 .

\subsection{Contamination Estimates}

One of the major sources of contamination in highredshift dropout galaxy samples is galaxies at $z \sim 2$ whose Balmer break mimics a Lyman- $\alpha$ break in the spectra of high- $z$ star-forming galaxies. Although bright $z \sim 2$ interlopers are removed by detections of a blue continuum in deep optical images, faint interlopers are selected due to the photometric uncertainties. Here we estimate the expected number of such contaminants that meet our dropout selection criteria, basically following the method described in Section 3.3 of Schenker et al. (2013, see also Section 3.1 of Ouchi et al. 2004).

To obtain the expected number of contaminants, we make use of our catalog of bright objects detected in the HFF fields. We assume that bright objects with $22<H_{160}<25$ that do not satisfy the dropout selection criteria are bright interlopers, and that faint interlopers have the color distribution same as that of bright ones. We create artificial objects with faint magnitudes of $H_{160}=25.0-29.5$ using the mkobjects package in IRAF (Tody 1986, 1993) software. Their number counts are matched to the observed number counts extrapolated from the bright magnitudes. These artificial objects are placed in random positions of our HFF images. We conduct the source extraction and the dropout selection in the same manner as our dropout galaxy identification in the real HFF data. The artificial objects selected as dropouts are regarded as contaminants. We derive 


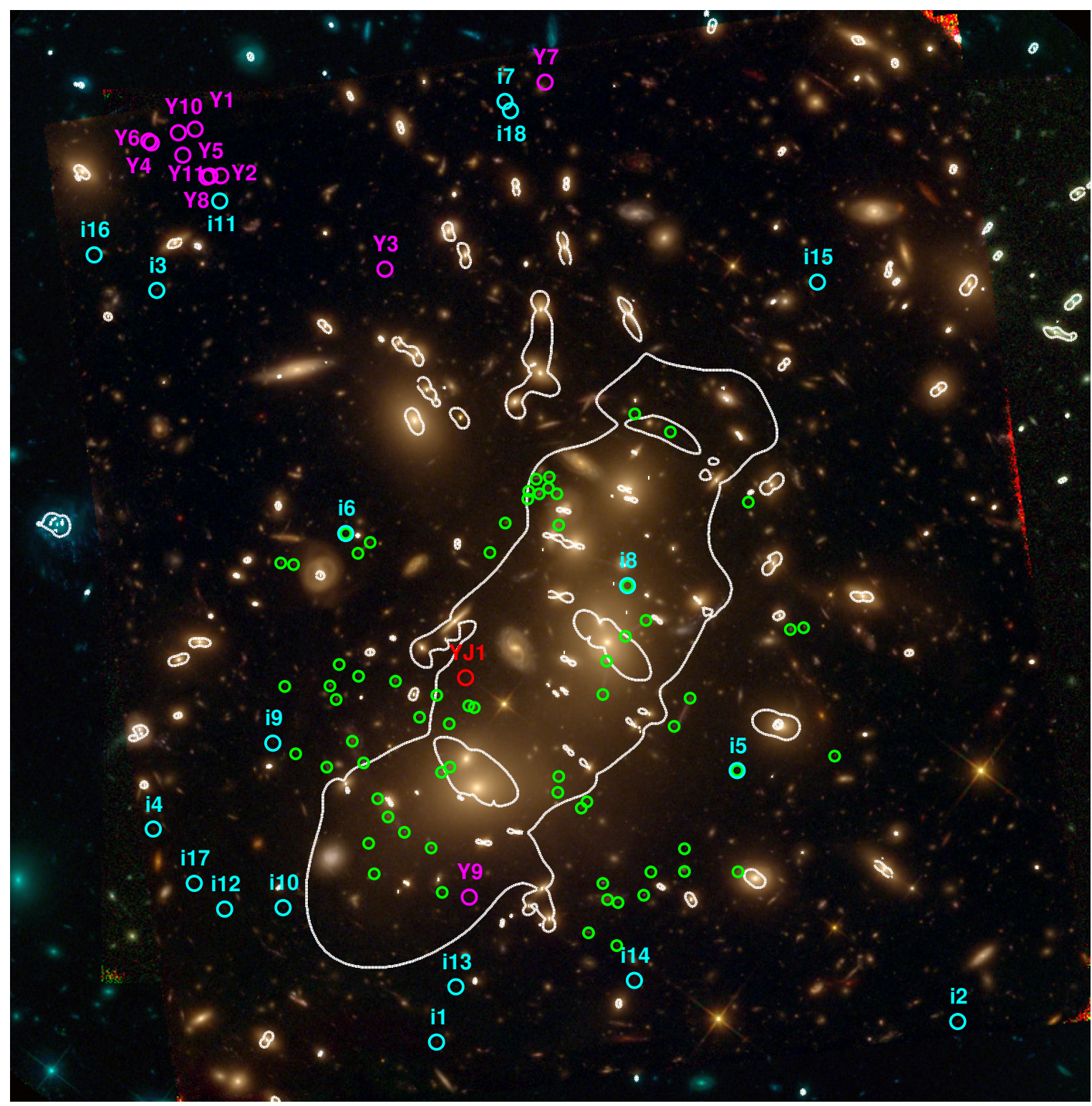

Figure 6. False-color image of the Abell 2744 cluster field (red: $J_{125}+J H_{140}+H_{160}$, green: $i_{814}+Y_{105}$, blue: $B_{435}+V_{606}$ ). The cyan, magenta, and red circles denote the positions of our dropout candidates at $z \sim 6-7, z \sim 8$, and $z \sim 9$, respectively. The green circles indicate the multiply imaged systems used for determination of our mass model. The critical lines for background sources at $z=8$ are shown with the white lines.

the numbers of contamination objects as a function of magnitude, which is used in our UV luminosity function estimates in Section 5.3 . We find that the fraction of total numbers of the contaminants to our dropout candidates down to 29.5 magnitude is $\sim 27 \%$. This fraction is relatively larger than the contamination rate estimated by some previous studies, which are for examples $\sim 7 \%$ at $z \sim 7-8$ in Bouwens et al. (2011) and $\sim 23 \%$ at $z \sim 9-10$ in Bouwens et al. (2012). In Section 6. we discuss the discrepancy between the UV luminosity densities and Thomson scattering optical depth. Because the differences of the number counts given by the contamination estimates are at most $30 \%$ which is smaller than the discrepancy discussed in Section 6, our conclusion does not change.
The gravitational lensing effects are important to interpret the observational results of our dropouts in the HFF fields. The brightness of dropouts are magnified, and multiple images appear for some of the dropouts. Thus, the number counts of our HFF dropout candidates are changed from those of the blank field by the gravitational lensing effects, especially in the cluster field. Moreover, to derive UV luminosity functions, one needs to correct for the selection incompleteness of dropouts that is a function of both magnitude and source redshift. In our study, we carry out Monte-Carlo simulations in the image plane to evaluate the gravitational lensing effects as well as the selection completeness. The simulations contain all lensing effects: magnification, distortion, and multiplication of images. This is called the image plane technique.

There is another method for the luminosity function estimates referred to as the source plane technique Atek

\subsection{Completeness Estimates}




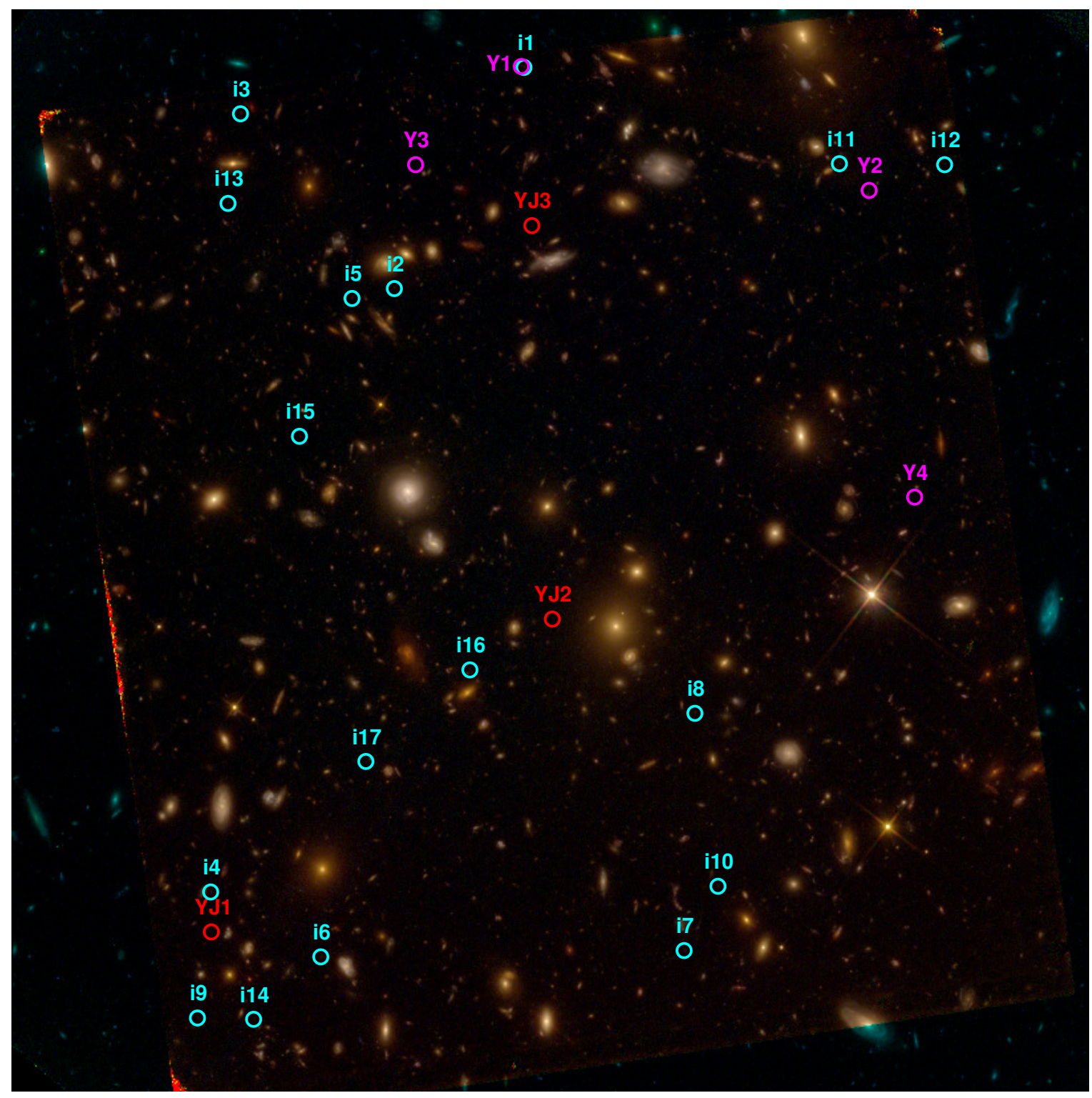

Figure 7. Same as Figure6 but for the Abell 2744 parallel field.

et al. 2014b). The source plane technique determines an absolute magnitude of each dropout candidate with a magnification factor to derive the number of dropout candidates per unit source plane volume. However, distortion and multiplication effects are not included in the source plane technique. Note that our method of the $\mathrm{im}$ age plane technique is self-consistent and more complete than the source plane technique.

We first estimate the completeness of dropouts identified by our selections in the HFF images, where the completeness depends on redshift and magnitude. We create a mock catalog of $\sim 1,000,000$ galaxies uniformly distributed at $z=5.0-10.4$ in the magnitude range of $25.0-30.5 \mathrm{mag}$. To define the UV continuum colors of the galaxies, we assume a spectral UV slope of $\beta=-2.0$, which is the same as that used in Schenker et al. (2013). IGM attenuation is given with the prescription given by Madau et al. (1996). For the galaxies' intrinsic surface brightness profiles, we adopt a Sérsic index of 1.0 and half-light radii of $\simeq 0.6 \mathrm{kpc}$ and $\simeq 0.3 \mathrm{kpc}$ for bright
$\left(M_{\mathrm{UV}} \lesssim-19.5 \mathrm{mag}\right)$ and faint $\left(M_{\mathrm{UV}} \gtrsim-19.5 \mathrm{mag}\right)$ dropout candidates, respectively, which are motivated by recent size measurements for $z \sim 7-8$ dropout candidates (Ono et al. 2013, see also, Oesch et al. 2010). We assume a uniform distribution of the intrinsic ellipticity in the range of $0.0-0.9$, because the observed ellipticities of $z \sim 3-5$ dropouts roughly have uniform distributions (Ravindranath et al. 2006).

Then, we produce simulated images of the galaxies that include the HST PSFs and the Abell 2744's gravitational lensing effects with writeimage command of GLAFIC. We randomly select about 3000 simulated images of galaxies in a magnitude bin of $\Delta m=0.5$, and place these simulated galaxy images at random positions on the real HFF images to make simulated HFF images. In the same manner as the procedure for the identifications of our real dropouts (Section 3.1), we perform source extractions for the simulated HFF images with SExtractor, and construct photometric catalogs. Applying the color selection criteria used in Section 3.2 and the 


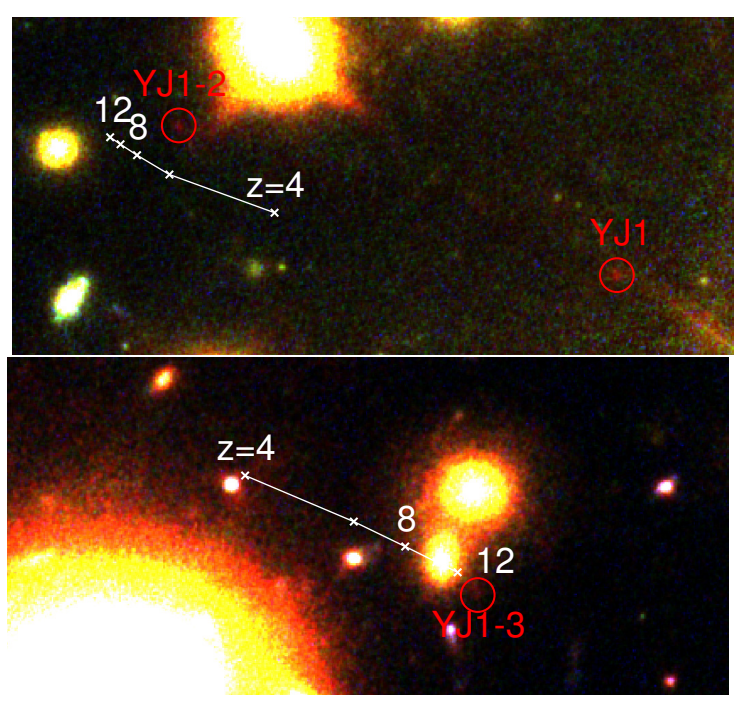

Figure 8. Predicted positions of multiple images of HFF1CYJ1 using our mass model. The red circles show the positions of HFF1C-YJ1 and its multiple images with the ID of HFF1C-YJ1-2 and HFF1C-YJ1-3. The radius of each circle is $0 . \prime 3$. The white crosses in the upper (lower) panel indicate the predicted position of image HFF1C-YJ1-2 (HFF1C-YJ1-3) at $z=4,6,8,10$, and 12 with the tracks over a redshift range $4<z<12$. Our mass model predicts that the redshift of YJ1 is $z>6$, as discussed in Zitrin et al. (2014).

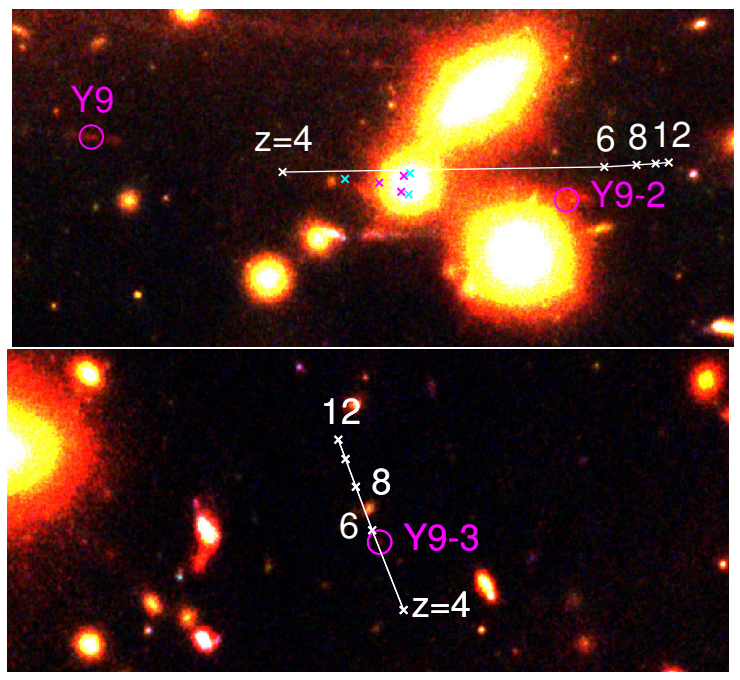

Figure 9. Same as Figure 8 but for HFF1C-Y9. The magenta circles show the positions of $\mathrm{HFF} 1 \mathrm{C}-\mathrm{Y} 9$ and its multiple images of HFF1C-Y9-2 and HFF1C-Y9-3. The cyan (magenta) crosses represent the positions of the other multiple images at $z=6(z=8)$ near a bright foreground galaxy, respectively.

magnitude-dependent contamination rates estimated in Section 5.1, we obtain simulated dropout galaxies. We make a simulated dropout galaxy sample for the magnitude bin. We conduct the same simulations over the given magnitude range of $25.0-30.5 \mathrm{mag}$, and derive the completeness for our $i$-, $Y$-, and $Y J$-dropout selections that depend on redshift and magnitude. Figure 12 shows the completeness derived from our simulations. The left (right) panels indicate the selection windows in the cluster (parallel) field. At bright magnitudes of $\sim 25-27$ mag, our $i-, Y$-, and $Y J$-dropout selection criteria provide a high completeness sample of star-forming galaxies at $6.0<z<7.4,7.4<z<8.6$, and $8.2<z<9.4$,

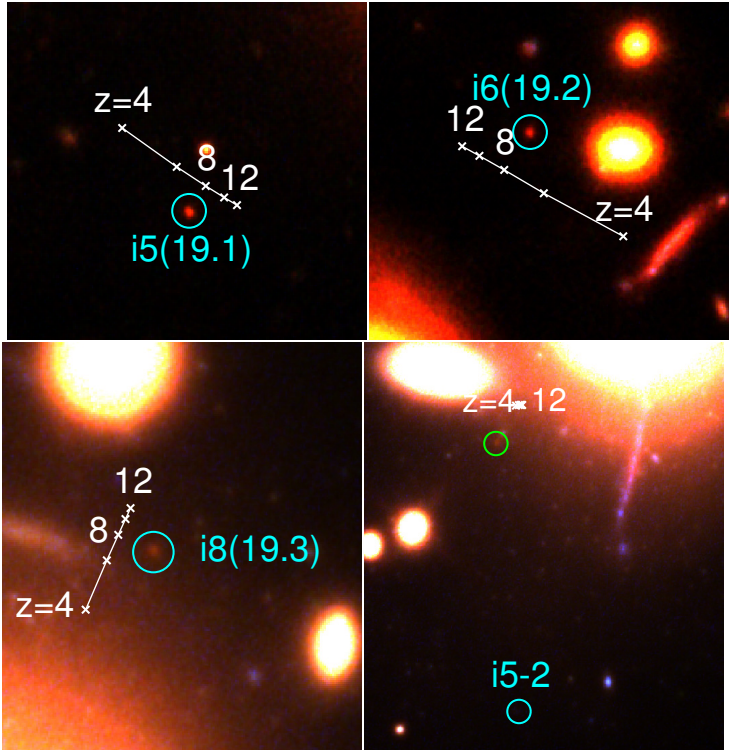

Figure 10. Same as Figure 8 but for HFF1C-i5, HFF1C-i6, and HFF1C-i8. The cyan circles show the positions of HFF1C-i5, HFF1C-i6, HFF1C-i8, and their multiple images. The green circle presents the position of $\mathrm{HFF} 1 \mathrm{C}-\mathrm{i} 5-2$ predicted by Jauzac et al. (2014).

respectively.

\subsection{Best-fit Schechter Parameters of UV Luminosity Functions}

These completeness estimates are then used to predict observed galaxy number counts. We calculate the predicted number counts from the completeness estimates and a UV luminosity function expressed as a Schechter function with a set of parameters, $\left(M_{*}, \phi_{*}, \alpha\right)$, and repeat it for various sets of Schechter parameters covering a wide parameter space. In this way, we obtain the predicted number counts for various sets of Schechter parameters. Because the completeness values are estimated with all of the observational effects in the image plane, these predicted number counts include the lensing magnifications, magnification, distortion, and multiplication as well as the corresponding detection incompleteness in the redshift and magnitude space.

Using the predicted number counts, we search for the best-fit Schechter parameters that reproduce the observed number counts of our dropout candidates. We adopt a maximum likelihood method, assuming a Poisson distribution of the number counts. The likelihood is written as

$$
\mathcal{L} \propto \prod_{\text {field }} \prod_{i} n_{\exp , i}^{n_{\mathrm{obs}, i}} e^{-n_{\exp , i}}
$$

where $n_{\exp , i}$ is the expected number counts from a given Schechter function in a magnitude interval $i$ and $n_{\mathrm{obs}, i}$ is the observed number counts in the magnitude interval. Constraining the Schechter parameters, we simultaneously fit both the observed HFF number counts and the UV luminosity function data points obtained in the previous studies. For our dropouts at $z \sim 6-7$, we compare our number counts of $z \sim 6-7$ with the luminosity function data points of $z \sim 7$ in previous studies, assuming that the UV luminosity function does not rapidly change in $z \sim 6-7$. We take the previous blank-field survey results from the studies of CANDELS, HUDF09, 

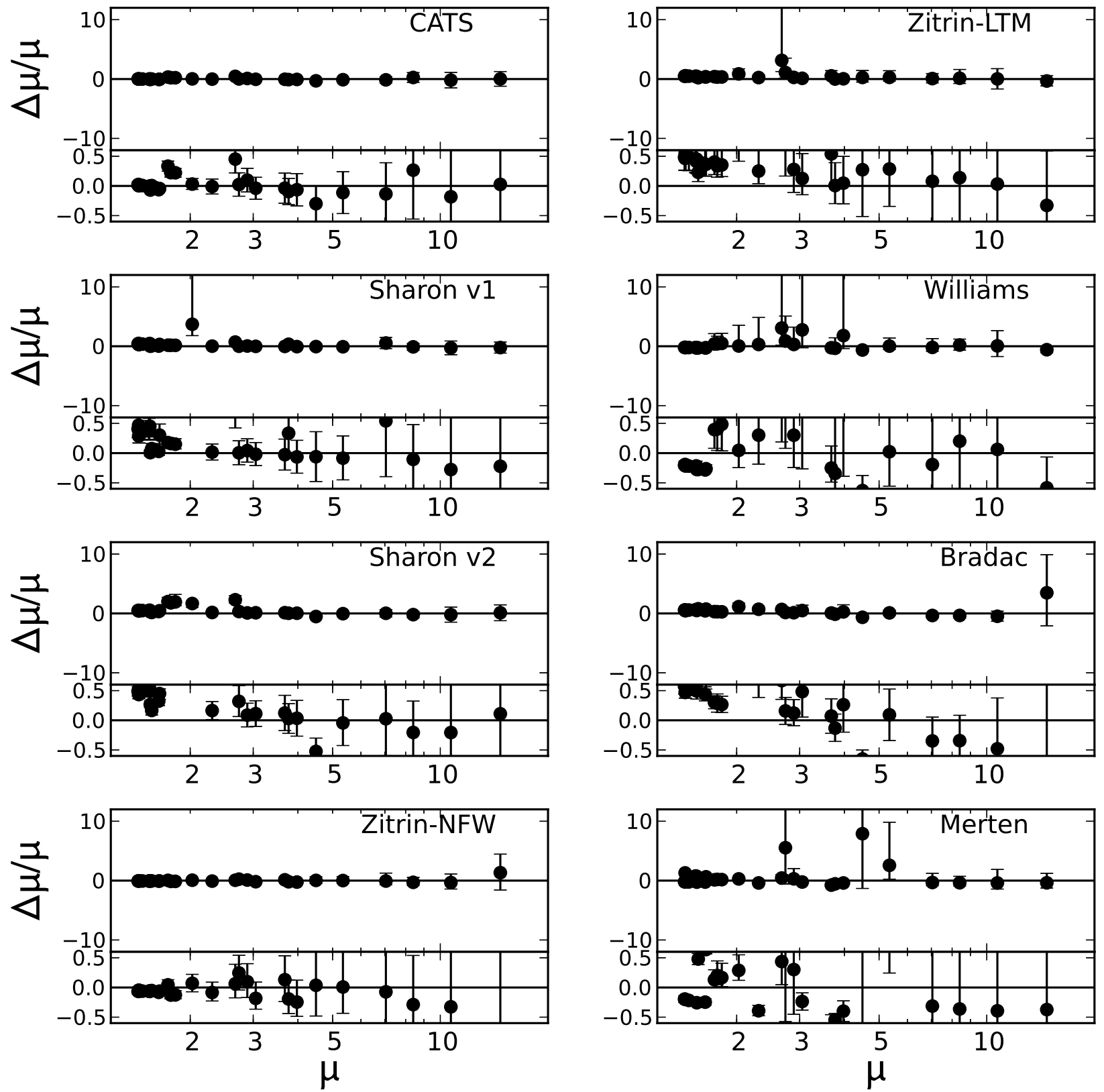

Figure 11. Comparison of the magnification factors of our dropouts for the different mass models in the cluster field. The horizontal axes show the magnification factor $\mu$ from our mass model, and the vertical axes present the difference $\Delta \mu$ between magnification factors of our model and a public model normalized by $\mu$. Eight panels present the public models of CATS, Sharon version 1 , Sharon version 2 , Zitrin-NFW, Zitrin-LTM, Williams, Bradač, and Merten. The upper and lower segments of each panel represent $\Delta \mu / \mu$ in the wide and narrow dynamic ranges, respectively.

HUDF12, ERS, and BORG/HIPPIES (Bouwens et al. 2014), UltraVISTA+UKIDSS UDS (Bowler et al. 2014), BoRG (Bradley et al. 2012), SDF+GOODS-N (Ouchi

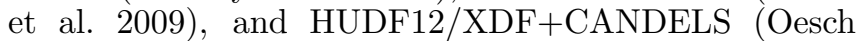
et al. 2013). We regard $M_{*}, \phi_{*}$, and $\alpha$ as free parameters for the fitting of number counts at $z \sim 6-7$ and $z \sim 8$. Because the statistics of the $z \sim 9$ luminosity function is poor, we choose $\phi_{*}$ for a free parameter and $M_{*}$ and $\alpha$ to be fixed to the best-fit values of $z \sim 8$. Maximizing the Poisson likelihood, we obtain the best-fit parameters of $\left(M_{*}, \log \phi_{*}\left[\mathrm{Mpc}^{-3}\right], \alpha\right)=$ $\left(-20.45_{-0.2}^{+0.1},-3.30_{-0.20}^{+0.10},-1.94_{-0.10}^{+0.09}\right)$

the $z^{-0.2} \sim 6-7^{-0.10}$ dropout candidates, $\left(-20.45_{-0.2}^{+0.3},-3.65_{-0.25}^{+0.15},-2.08_{-0.12}^{+0.21}\right)$ for the $z \sim 8$ dropout candidates, and $(-20.45[$ fixed], $-4.00 \pm$ $0.15,-2.08$ [fixed]) for the $z \sim 9$ dropout candidates. Table 7 summarizes these parameters, together with those obtained by the previous studies. We find that 


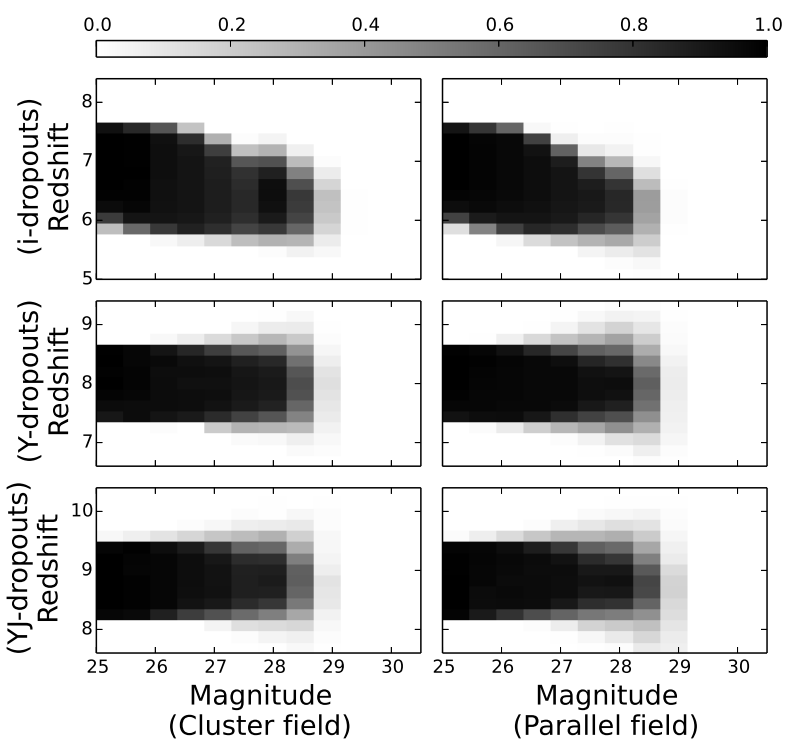

Figure 12. Completeness of our dropout selections that depend on redshift and apparent magnitude in the cluster (left) and parallel (right) fields. The top, middle, and bottom panels are the completeness of $i-, Y$, and $Y J$-dropout candidates, respectively. The completeness values are normalized to 1.0 at the maximum completeness values in the selection. The darker shades indicate the higher completeness.

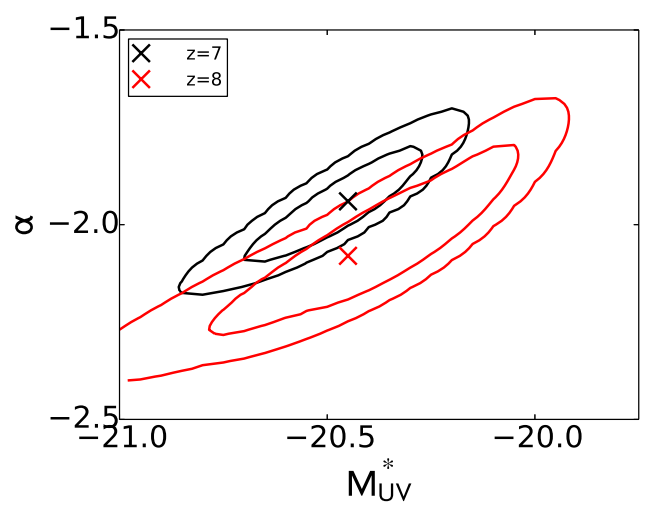

Figure 13. The $68 \%$ and $95 \%$ confidence level contours of Schechter parameters, $M_{*}$ and $\alpha$, for the UV luminosity functions at $z \sim 6-7$ (black) and $z \sim 8$ (red), respectively. The contours indicate our best-estimate results with our HFF data and all of the previous measurements. The crosses denote the best-fit parameter values.

our results are consistent with the previous results within the $1 \sigma$ uncertainties. Figures 13 and show the $1 \sigma$ confidence intervals on the $\alpha$ versus $\bar{M}_{*}$ plane for the UV luminosity functions at $z \sim 6-7$ and $z \sim 8$, respectively. To test our results, we also perform Schechter function fittings without the results from the HUDF09+ERS data at $z \sim 6-7$ and $z \sim 8$. We confirm that the fitting results without the HUDF09+ERS data are consistent with the previous results, although the uncertainties are substantially large due to the small statistics of the HFF samples.

The top and bottom panels of Figures $[14,16$ present
Table 7

Best-fit Schechter parameters of luminosity functions

\begin{tabular}{|c|c|c|c|}
\hline Reference & $M_{*}$ & $\log \phi_{*}\left[\mathrm{Mpc}^{-3}\right]$ & $\alpha$ \\
\hline \multicolumn{4}{|l|}{$z \sim 6-7$} \\
\hline This Work & $-20.45_{-0.2}^{+0.1}$ & $-3.30_{-0.20}^{+0.10}$ & $-1.94_{-0.10}^{+0.09}$ \\
\hline Atek et al. (2014a) & $-20.63_{-0.56}^{+0.69}$ & $-3.34 \pm 0.36$ & $-1.88_{-0.20}^{+0.17}$ \\
\hline Bouwens et al. (2014) & $-21.04 \pm 0.26$ & $-3.65_{-0.17}^{+0.27}$ & $-2.06 \pm 0.12$ \\
\hline Bouwens et al. (2011) & $-20.14 \pm 0.26$ & $-3.07 \pm 0.26$ & $-2.01 \pm 0.21$ \\
\hline Ouchi et al. (2009) & $-20.10 \pm 0.76$ & $-3.16 \pm 0.68$ & $-1.72 \pm 0.65$ \\
\hline Schenker et al. (2013) & $-20.14_{-0.48}^{+0.36}$ & $-3.19_{-0.24}^{+0.27}$ & $-1.87_{-0.17}^{+0.18}$ \\
\hline \multicolumn{4}{|l|}{$z \sim 8$} \\
\hline This Work & $-20.45_{-0.2}^{+0.3}$ & $-3.65_{-0.25}^{+0.15}$ & $-2.08_{-0.12}^{+0.21}$ \\
\hline Bouwens et al. 2014 & $-19.97 \pm 0.34$ & $-3.19 \pm 0.30$ & $-1.86 \pm 0.27$ \\
\hline Bouwens et al. $(2011)$ & $-20.10 \pm 0.52$ & $-3.22 \pm 0.43$ & $-1.91 \pm 0.32$ \\
\hline Bradley et al. $(2012)$ & $-20.26_{-0.34}^{+0.29}$ & $-3.37_{-0.29}^{+0.26}$ & $-1.98_{-0.22}^{+0.23}$ \\
\hline $\begin{array}{ll}\text { Schenker et al. } & (2013) \\
\end{array}$ & $-20.44_{-0.35}^{+0.47}$ & $-3.50_{-0.32}^{+0.35}$ & $-1.94_{-0.24}^{+0.21}$ \\
\hline \multicolumn{4}{|l|}{$z \sim 9$} \\
\hline This Work & -20.45 (fixed) & $-4.00 \pm 0.15$ & -2.08 (fixed) \\
\hline Oesch et al. (2013) & $-18.8 \pm 0.3$ & -2.94 (fixed) & -1.73 (fixed) \\
\hline Bouwens et al. $(2012)$ & -20.04 (fixed) & $-3.95_{-0.56}^{+0.39}$ & -2.06 (fixed) \\
\hline
\end{tabular}

the best-fitting number counts and Schechter functions, respectively. In the bottom panels, we present recent studies of $z \sim 7$ and 8 luminosity functions, Schenker et al. (2013), Finkelstein et al. (2014), and Atek et al. (2014a) for comparison. The best-fitting results broadly agree with the observed number counts. The observed number counts of $z \sim 8$ at the bright end are larger than the best-fit function. It is probably caused by the fieldto-field variance, since our effective survey area is only $\simeq 6 \operatorname{arcmin}^{2}$ in the source plane. In fact, eight of the $z \sim 8$ dropouts are found within a small region with a radius of $6^{\prime \prime}$ (corresponding to a physical length of $\sim 30$ $\mathrm{kpc}$ at $z=8$ ). This overdensity of $z \sim 8$ dropouts is originally claimed by Zheng et al. (2014) with the early optical images shallower than our full-depth data by $\sim 1$ magnitudes. Because one cannot remove a number of foreground interlopers with the shallow early optical data, the existence of overdensity is open question (see the discussion in Coe et al. 2014). In our study with the full-depth HFF data deep enough to remove such foreground interlopers reliably, there is the overdensity of $z \sim 8$ dropouts, indicative that the overdensity is real. If it is true, the existence of the overdensity would significantly enhance the source number counts of dropouts at $z \sim 8$ in the HFF fields.

The middle panels of Figures 14, 16 show the histograms of the numbers of dropout galaxies used for the UV luminosity function determinations. We also show the numbers of dropout galaxies newly identified in the observations of the HDF12 at $z \sim 7-8$ (Schenker et al. 2013) and those of CLASH at $z \sim 9.2$ (Bouwens et al. 2012). These histograms indicate that our HFF' samples enable us to probe the UV luminosity functions down to a faint UV magnitude of $\simeq-17$, which is comparable to survey limits of the deepest blank-field observations of the HUDF (Ellis et al. 2013: Oesch et al. 2013), thanks to the gravitational lensing effects. In addition to the lensing effects, the HFF Abell 2744 observations provide two new ultra-deep imaging regions of the cluster and parallel fields, allowing us to significantly increase the number of 

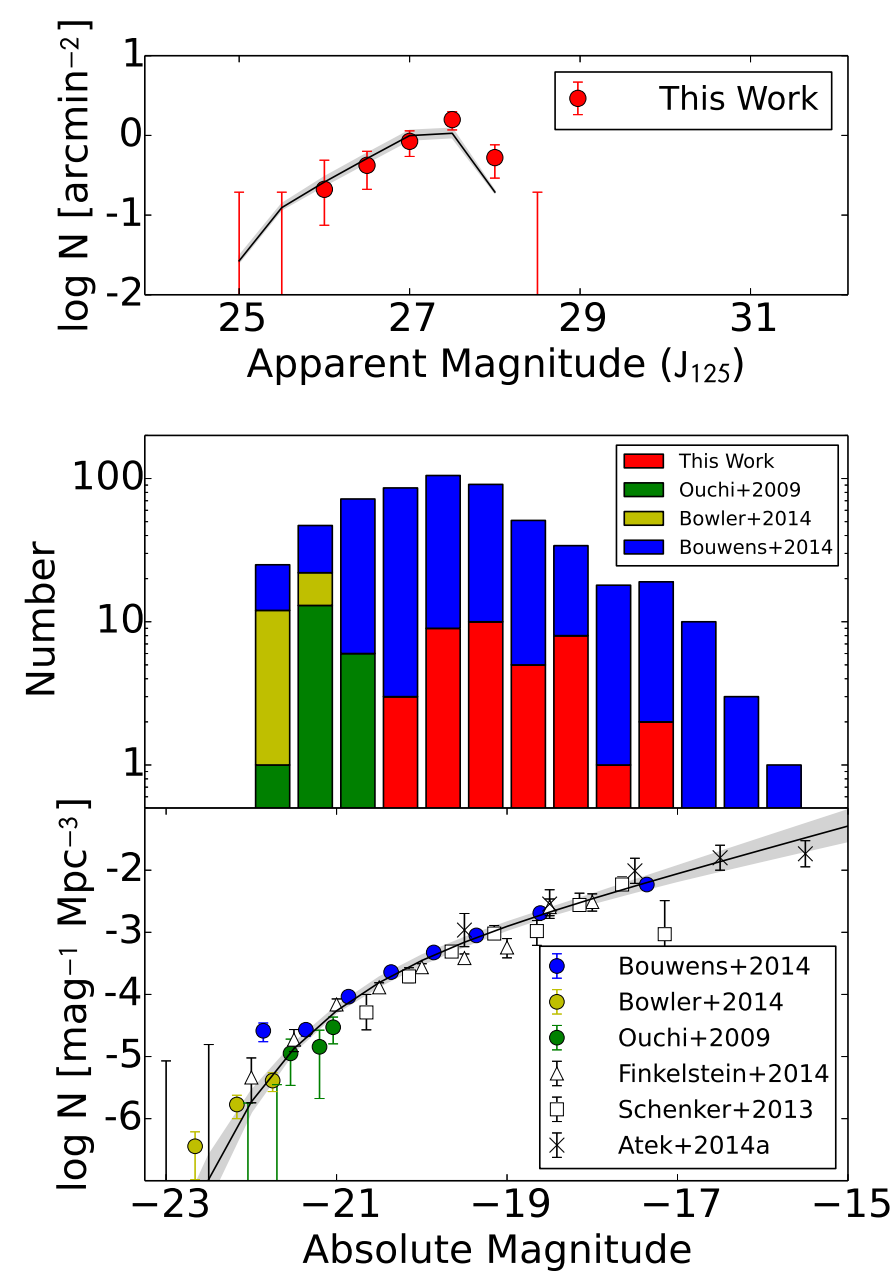

Figure 14. Number counts, histograms, and luminosity functions of $z \sim 6-7$ dropouts. Top panel: Our observed number counts in the cluster and parallel fields (red circles) and the simulated number counts of the best-fit Schechter parameters (black line) with the $1 \sigma$ uncertainties (gray region). The horizontal axis presents observed apparent magnitude in the $J_{125}$ band. Middle panel: The histograms of the numbers of dropouts found in our HFF study (red) and the previous work, Bouwens et al. (2014) (blue), Ouchi et al. (2009) (green), and Bowler et al. (2014) (yellow). Bottom panel: Our best-fit luminosity function (black line) and the $1 \sigma$ error (gray region). The blue, green, and yellow circles, white triangles, white squares, and black crosses denote luminosity functions derived by Bouwens et al. (2014), Ouchi et al. (2009), Bowler et al. (2014), Finkelstein et al. (2014), Schenker et al. (2013), and Atek et al. (2014a), respectively. The horizontal axis shows intrinsic absolute magnitude in the $J_{125}$ band.

$z \sim 9$ dropouts (cf. the $z \sim 9$ dropout samples in UDF12 and CANDELS Oesch et al. 2013).

\section{DISCUSSION}

In Section 5, we have derived the UV luminosity functions of dropout galaxies at $z \sim 6-7,8$, and 9 based on our HFF and the previous study data. We improve the faint-end luminosity function determinations with the large samples extending the magnitude range. The UV luminosity functions are tightly connected with the production rates of ionizing photons escaping to the IGM, which are important observational quantities to under-
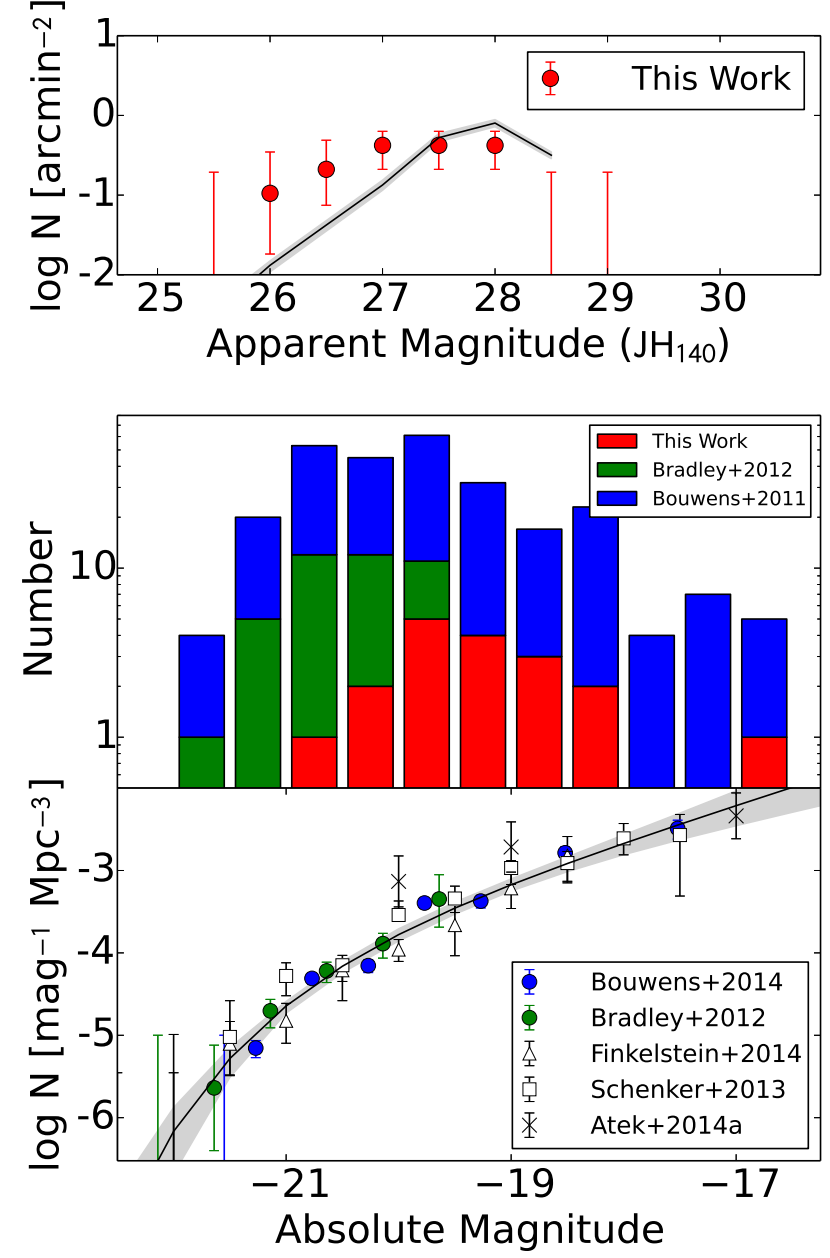

Figure 15. Same as Figure 14 but for $z \sim 8$. The horizontal axes in the top and bottom panels present apparent magnitude and intrinsic absolute magnitude in the $J H_{140}$ band, respectively. We show the results of Bradley et al. (2012) with the green histogram and circles in the middle and bottom panels, respectively.

stand the process of cosmic reionization, In this section, we carry out the joint analysis of the UV luminosity functions and the electron scattering optical depth $\tau_{e}$ measured by the CMB observations to discuss the ionizing sources of the IGM.

\subsection{Evolution of the UV Luminosity Density}

To investigate the ionizing sources for the cosmic reionization, we first estimate UV luminosity densities $\rho_{\mathrm{UV}}$ from our UV luminosity functions. $\rho_{\mathrm{UV}}$ is calculated by

$$
\rho_{\mathrm{UV}}(z)=\int_{-\infty}^{M_{\mathrm{trunc}}} \Phi\left(M_{\mathrm{UV}}\right) L\left(M_{\mathrm{UV}}\right) d M_{\mathrm{UV}}
$$

where $M_{\text {trunc }}$ is the truncation magnitude of the UV luminosity function where no galaxies exist beyond this magnitude. Because the $M_{\text {trunc }}$ parameter is not constrained by observations, in this study we assume two $M_{\text {trunc }}$ values that bracket the plausible range of the parameter; $M_{\text {trunc }}=-17$ mag corresponding to the current 

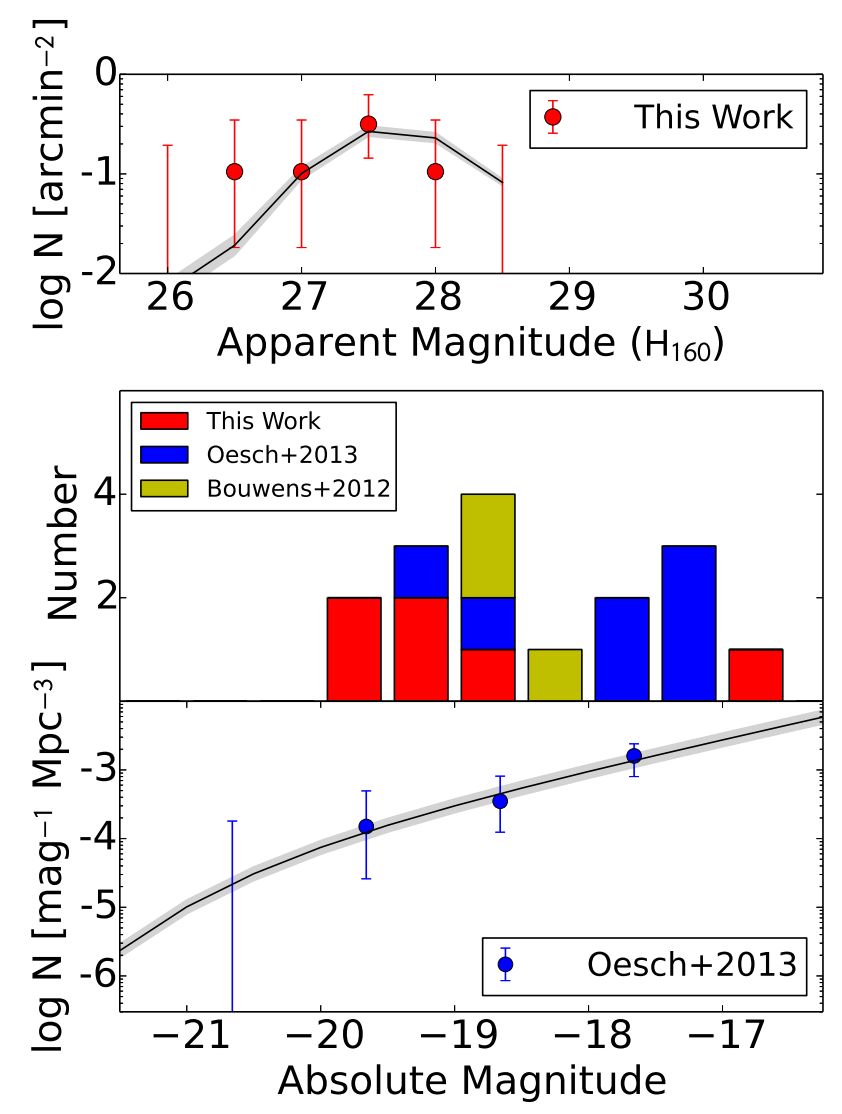

Figure 16. Same as Figure 14 but for $z \sim 9$. The horizontal axes in the top and bottom panels present apparent magnitude and intrinsic absolute magnitude in the $H_{160}$ band, respectively. The yellow histogram shows the number of dropouts found in Bouwens et al. (2012). The blue histogram and circles are the numbers and luminosity functions, respectively, obtained by Oesch et al. (2013).

observational limit and $M_{\text {trunc }}=-10$ mag being the predicted magnitude of minimum-mass halos that can host star-forming galaxies (Faucher-Giguère et al. 2011).

We use the best-fit Schechter functions shown in Section 5 and those in the literature for $z \sim 4-6$ (Bouwens et al. 2007), $z \sim 9.2$ (Bouwens et al. 2012), and $z \sim 10.4$ (Bouwens et al. 2014). For comparison purpose, we plot the data for $z \sim 7-8$ taken from Schenker et al. (2013) and McLure et al. (2013). Because the data of Schenker et al. (2013) and McLure et al. (2013) are included in our luminosity function estimates via Bouwens et al. (2014) data points, we do not use these data points of Schenker et al. (2013) and McLure et al. (2013) for fitting analyses carried out in Section 6.2 'The top and bottom left panels of Figure 17present the $\rho_{\mathrm{UV}}$ as a function of redshift under the assumptions of $M_{\text {trunc }}=-17$ and -10 , respectively. The solid and dashed lines show the bestfit functions of $\rho_{\mathrm{UV}}$ with two fitting methods detailed in Section 6.2. We confirm that our $\rho_{\mathrm{UV}}$ at $z \sim 6-9$ are broadly consistent with the previous results, and that there is a rapid decrease of $\rho_{\mathrm{UV}}$ from $z \sim 8$ towards high redshifts, which is claimed by Oesch et al. (2013) and Bouwens et al. (2014). With the improved measurements of $\rho_{\mathrm{UV}}$ in our study, this trend of the rapid decrease is strengthened. To test whether the rapid decrease is confirmed with the HFF data alone, we derive the luminosity function at $z \sim 9$ with the HFF data alone, and estimate $\rho_{\mathrm{UV}}$ at $z \sim 9$. The gray circles in the left panels of Figure 17 indicate $\rho_{\mathrm{UV}}$ obtained with our HFF data alone. Although the uncertainty is large, the HFF data independently support the rapid decrease of $\rho_{\mathrm{UV}}$ from $z \sim 8$. Similar analysis is found in Oesch et al. (2014). They derive the luminosity function at $z \sim 10$ from the HFF cluster data alone. we plot $\rho_{\mathrm{UV}}$ at $z \sim 10$ calculated from the luminosity function derived by Oesch et al. (2014) with the gray squares. These plots are also consistent with the rapid decrease from $z \sim 8$.

Robertson et al. (2014) estimate the cosmic variance uncertainties of the high-redshift galaxies in the Abell 2744 cluster field. The uncertainties are $\sim 35 \%$ at $z \sim$ 7 and $\gtrsim 65 \%$ at $z \sim 10$. Our errors of $\rho_{\mathrm{UV}}$ slightly increase by the cosmic variance uncertainties. However, our conclusion does not change because our $\rho_{\text {UV }}$ at $z \sim 9$ is smaller than $\rho_{\mathrm{UV}}$ at $z \sim 8$ by a factor of two, which is significantly larger than the uncertainties of the cosmic variance.

\subsection{Properties of the Ionizing Sources Revealed from the $\rho_{\mathrm{UV}}$ and $\tau_{e}$ Measurements}

The evolution of the ionized hydrogen fraction in the IGM, $Q_{\mathrm{H}_{\mathrm{II}}}$, is described by the following ionization equation (e.g., Robertson et al. 2013),

$$
\dot{Q}_{\mathrm{H}_{\mathrm{II}}}=\frac{\dot{n}_{\mathrm{ion}}}{\left\langle n_{\mathrm{H}}\right\rangle}-\frac{Q_{\mathrm{H}_{\mathrm{II}}}}{t_{\mathrm{rec}}}
$$

where the dots denote time derivatives.

The first term in the right-hand side of Equation (31) is a source term proportional to the ionizing photon emissivity. $\dot{n}_{\text {ion }}$ and $\left\langle n_{\mathrm{H}}\right\rangle$ are the production rate of ionizing photons and the mean hydrogen number density, respectively. They are defined by

$$
\begin{aligned}
\dot{n}_{\text {ion }} & =\int_{-\infty}^{M_{\text {trunc }}} f_{\text {esc }}\left(M_{\mathrm{UV}}\right) \xi_{\text {ion }}\left(M_{\mathrm{UV}}\right) \Phi\left(M_{\mathrm{UV}}\right) L\left(M_{\mathrm{UV}}\right) d M_{\mathrm{UV}} \\
& \equiv\left\langle f_{\mathrm{esc}} \xi_{\text {ion }}\right\rangle \rho_{\mathrm{UV}} \\
\left\langle n_{\mathrm{H}}\right\rangle & =\frac{X_{\mathrm{p}} \Omega_{\mathrm{b}} \rho_{\mathrm{c}}}{m_{\mathrm{H}}} .
\end{aligned}
$$

$X_{\mathrm{p}}$ is the primordial mass fraction of hydrogen, $\rho_{\mathrm{c}}$ is the critical density, and $m_{\mathrm{H}}$ is the mass of the hydrogen atom. Note that $f_{\text {esc }}$ and $\xi_{\text {ion }}$ are parameters that appear in the product form for our analysis. If one assumes that $f_{\text {esc }}$ and $\xi_{\text {ion }}$ depend on $M_{\mathrm{UV}},\left\langle f_{\text {esc }} \xi_{\text {ion }}\right\rangle$ is a magnitudeaveraged value defined in Equation 32 .

The second term in the right-hand side of Equation (31) is a sink term due to recombinations; $t_{\mathrm{rec}}$ is the averaged gas recombination time,

$$
t_{\mathrm{rec}}=\frac{1}{C_{\mathrm{H}_{\mathrm{II}}} \alpha_{\mathrm{B}}(T)\left(1+Y_{\mathrm{p}} / 4 X_{\mathrm{p}}\right)\left\langle n_{\mathrm{H}}\right\rangle(1+z)^{3}},
$$

where $\alpha_{\mathrm{B}}$ is the case $\mathrm{B}$ hydrogen recombination coefficient, and $T$ is the IGM temperature at a mean density. $Y_{\mathrm{p}}$ is the primordial helium mass fraction. Substituting 

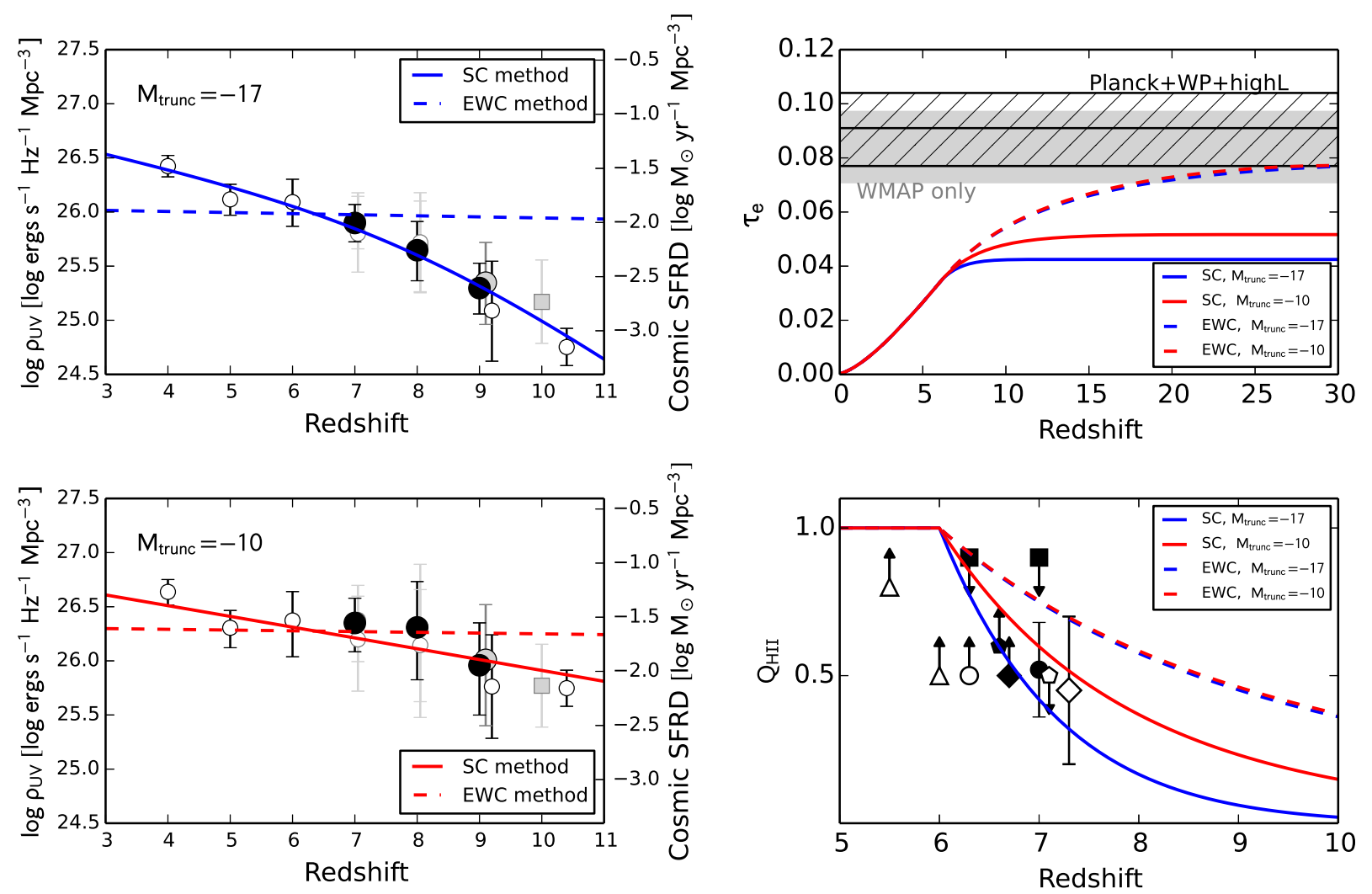

Figure 17. Upper left panel: The UV luminosity densities calculated with $M_{\text {trunc }}=-17$. The filled and open circles represent the UV luminosity densities from this work and other studies (Bouwens et al. 2007 Schenker et al. 2013 McLure et al. 2013 Bouwens et al. 2012. 2014), respectively. The gray circles and squares denote the UV luminosity densities from the HFF data only in this work and in Oesch et a1. (2014), respectively. The solid and dashed lines present our best-fit functions of $\rho_{\mathrm{UV}}$ with the SC and the EWC method, respectively. The right axes show cosmic SFR densities at a given UV luminosity density estimated with the Equation (2) of Madau et al. (1998). Bottom left panel: Same as the top left panel, but for $M_{\text {trunc }}=-10$. Upper right panel: Electron scattering optical depth integrating trom $z \sim 0$ to a redshift, $z$, for our best-fit parameters by the SC method with $M_{\text {trunc }}=-17$ (blue solid line), the EWC method with $M_{\text {trunc }}=-10$ (red solid line), the EWC method with $M_{\text {trunc }}=-17$ (blue dashed line), and the EWC method with $M_{\text {trunc }}=-10$ (red dashed line), respectively. The hatched and gray regions indicate the $1 \sigma$ range of $\tau_{e}$ obtained by WMAP+Planck + highL (Planck Collaboration et al. 2013 ) and nine-year WMAP (Hinshaw et al. 2013 Bennett et al. 2013), respectively. Bottom right panel: The evolution of ionized hydrogen fraction $Q_{\mathrm{H}_{\mathrm{II}}}$ of IGM for our best-fit parameters with four lines, whose notations are the same as the right upper panel. Each symbol represents the observational limit of Bolton et al. (2011), Carilli et al. (2010) (filled squares), Totani et al. (2006), McQuinn et al. (2008) (open circle), McQuinn et al. (2007), Ouchi et al. (2010) (filled diamond), Ouchi et al. (2010), Ota et al. (2008) (open pentagon), Mesinger (2010), McGreer et al. (2011) (open triangles), McQuinn et al. (2007), Mesinger \& Furlanetto (2008), Dijkstra et al. (2011) (filled pentagon), and Konno et al. (2014) (open diamond) (see also Robertson et al. 2013).

Equations (32)-(34) into Equation (31), we obtain

$$
\begin{aligned}
\dot{Q}_{\mathrm{H}_{\mathrm{II}}} & =A\left(\frac{\rho_{U V}}{10^{26} \mathrm{ergs} \mathrm{s}^{-1} \mathrm{~Hz}^{-1} \mathrm{Mpc}^{-3}}\right)-\frac{Q_{\mathrm{H}_{\mathrm{II}}}}{t_{\mathrm{rec}}} \\
A & =2.06 \mathrm{Gyr}^{-1}\left(\frac{\left\langle f_{\mathrm{esc}} \xi_{\text {ion }}\right\rangle}{0.2 \times 10^{25.2} \mathrm{erg} \mathrm{Hz}^{-1}}\right) \\
t_{\mathrm{rec}} & =3.19 \times 10^{2} \mathrm{Gyr}(1+z)^{-3}\left(\frac{C_{\mathrm{H}_{\mathrm{II}}}}{3}\right)^{-1} .
\end{aligned}
$$

Once the evolution of $Q_{\mathrm{H}_{\mathrm{II}}}$ is determined by these equations, $\tau_{e}$ at a redshift $z$ is estimated (e.g., Kuhlen \& Faucher-Giguère 2012) from

$$
\begin{aligned}
\tau_{e}(z)=\int_{0}^{z} \frac{c\left(1+z^{\prime}\right)^{2}}{H\left(z^{\prime}\right)} Q_{\mathrm{H}_{\mathrm{II}}} \sigma_{\mathrm{T}}\left\langle n_{\mathrm{H}}\right\rangle \\
\times\left(1+\eta Y_{\mathrm{p}} / 4 X_{\mathrm{p}}\right) d z^{\prime},
\end{aligned}
$$

where $c$ is the speed of light, $H(z)$ is the Hubble parameter, and $\sigma_{\mathrm{T}}$ is the Thomson scattering cross section. We assume that helium is singly ionized $(\eta=1)$ at $z>4$ and doubly ionized $(\eta=2)$ at $z<4$ (Kuhlen \& Faucher-Giguère 2012). The value of $\tau_{e}$ is measured to be $\tau_{e}=0.091_{-0.014}^{+0.013}$ (Planck Collaboration et al. 2013) from the combination of the Planck temperature power spectrum, the WMAP polarization low-multipole $(l \leq 23)$ likelihood (Bennett et al. 2013), and the high-resolution ground-based CMB data (e.g., Reichardt et al. 2012 , Story et al. 2013).

We assume that $\rho_{\mathrm{UV}}$ is approximated by a logarithmic double power law,

$$
\rho_{\mathrm{UV}}(z)=\frac{2 \rho_{\mathrm{UV}, z=8}}{10^{a(z-8)}+10^{b(z-8)}}
$$

where $\rho_{\mathrm{UV}, z=8}$ is a normalization factor, and $a$ and $b$ determine the slopes of $\rho_{\mathrm{UV}}(z)$. This double power-law function recovers the rapid decrease of $\rho_{\mathrm{UV}}$ from $z \sim 8$ towards high redshifts.

With the analytic reionization models described with Equations $35-39$, we carry out $\chi^{2}$ fitting to the observational data of $\tau_{e}$ and $\rho_{\mathrm{UV}}$ to search for reioniza- 
tion models allowed by these observational constraints. There are six free parameters in the fit, $\rho_{\mathrm{UV}, z=8}, a, b$, $\left\langle f_{\text {esc }} \xi_{\text {ion }}\right\rangle$, and $C_{\mathrm{H}_{\text {II }}}$. Because there is no observational data point of $\rho_{\mathrm{UV}}$ at $z>11$, we extrapolate the best-fit $\rho_{\mathrm{UV}}$ function of $z<11$ to $z=30$. At $z>30$, we assume $\rho_{\mathrm{UV}}=0$. In conjunction with this assumption, we regard that $\tau_{e}(z=30)$ should agree with the $\tau_{e}$ value from the CMB measurements.

For the data of $\rho_{\mathrm{UV}}$ in the fitting, we use all of the $\rho_{\mathrm{UV}}$ data points presented in Figure 17. (Section 6.1), except for those given by Schenker et al. (2013) and McLure et al. (2013) at $z=7$ and 8. Note that the data from these two studies are already included in our $\rho_{\mathrm{UV}}$ estimates via our best-fit UV luminosity functions in Section 5.3 We, thus, use a total of $8 \rho_{\mathrm{UV}}$ data points for the fitting.

The fitting ranges of $C_{\mathrm{H}_{\mathrm{II}}}$ and $\left\langle f_{\text {esc }} \xi_{\text {ion }}\right\rangle$ are $1.0-9.9$ and $0-10^{25.2} \mathrm{erg}^{-1} \mathrm{~Hz}$, respectively. The range of $\left\langle f_{\text {esc }} \xi_{\text {ion }}\right\rangle$ is motivated by the estimate of spectral properties of high-redshift galaxies in Robertson et al. (2013). We calculate the $\chi^{2}$ value by simply summing up the $\chi^{2}$ value of each data point of $\rho_{\mathrm{UV}}$ and $\tau_{e}$, and obtain the best-fitting parameters. We refer to this fitting method as a simple $\chi^{2}(\mathrm{SC})$ method.

From the $\chi^{2}$ minimization of the SC method, we find the best-fit parameters for $M_{\text {trunc }}=-17$ and -10 . The best-fit parameters and the $\chi^{2}$ values are shown in Table 8. Figure 18 presents $\Delta \chi^{2}$ values on the $\left\langle f_{\text {esc }} \xi_{\text {ion }}\right\rangle$ versus $C_{\mathrm{H}_{\text {II }}}$ plane calculated by the SC method. $\Delta \chi^{2}$ is determined by $\Delta \chi^{2} \equiv \chi^{2}-\chi_{\min }^{2}$, where $\chi_{\min }^{2}$ is the minimum $\chi^{2}$ value. In Figure 17, we show the best-fit functions of $\rho_{\mathrm{UV}}(z)$ and $\tau_{e}(z)$ for $M_{\text {trunc }}=-17$ and -10 . Figure 17 indicates that the best-fit $\rho_{\mathrm{UV}}(z)$ agrees with the data points, but that the best-fit $\tau_{e}$ is significantly lower than the one of the CMB measurement. The $\chi^{2}$ values and the degrees of freedom (dof) shown in Table 8 suggest that the probabilities of these $\chi^{2}$ values occurring by chance are $0.4 \%$ and $1.8 \%$ with $M_{\text {trunc }}=-17$ and -10 , respectively. It indicates that our analytic reionization models may not be good enough to explain the reionization history and sources of reionization.

The best-fit functions by the SC method are weighted by the $\rho_{\mathrm{UV}}$ data more strongly than the $\tau_{e}(z)$ data, because the number of data points of $\rho_{\mathrm{UV}}$ is 8 , while that of $\tau_{e}(z)$ is 1 . Here, we calculate $\chi^{2}$ values by another method which gives equal weight to $\rho_{\mathrm{UV}}$ and $\tau_{e}$ data sets. In this method, we divide the $\chi^{2}$ values of the $\rho_{\mathrm{UV}}$ data by 8 , that is the number of the data points. We refer to this method as an equally-weighted $\chi^{2}$ (EWC) method. The best-fit parameters and functions by the EWC method are shown in Table 8 and Figure 17, respectively. In the case of EWC, the best-fit $\tau_{e}$ falls in the error range of the CMB measurement as we expect. However, there is a discrepancy between the best-fit $\rho_{\mathrm{UV}}(z)$ and the observational $\rho_{\mathrm{UV}}$ data points especially at $z \gtrsim 9$ where the observational $\rho_{\mathrm{UV}}$ data exhibit the rapid decrease towards high- $z$.

Even if we change the weights of the fitting and allow the large parameter space of $\left\langle f_{\text {esc }} \xi_{\text {ion }}\right\rangle$ and $C_{\mathrm{H}_{\mathrm{II}}}$, we have found that no single model can reproduce both the $\tau_{e}$ and $\rho_{\mathrm{UV}}$ data points from the observations. This is because the data points of $\rho_{\mathrm{UV}}$ decrease too rapidly at $z>8$ to contribute to adding $\tau_{e}$. This conclusion is
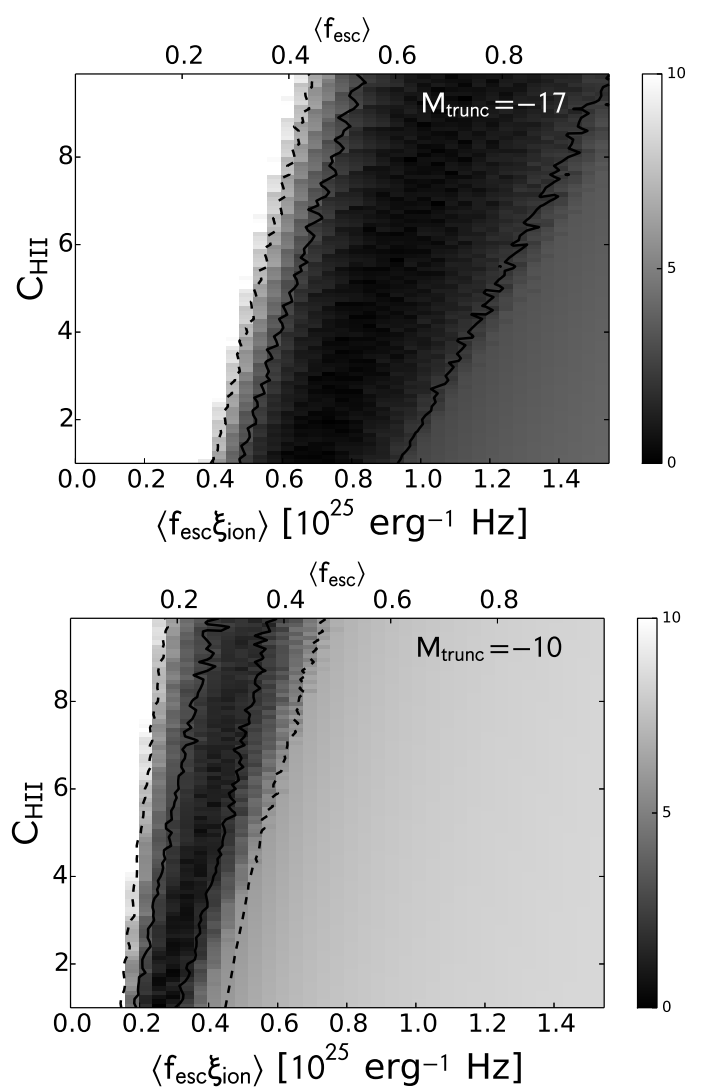

Figure 18. $\Delta \chi^{2}$ maps and confidence contours of $C_{\mathrm{H}_{\mathrm{II}}}$ and $\left\langle f_{\text {esc }} \xi_{\text {ion }}\right\rangle$ by the SC method with $M_{\text {trunc }}=-17$ (upper panel) and $M_{\text {trunc }}=-10$ (bottom panel). The upper axes indicate the average escape fraction $\left\langle f_{\text {esc }}\right\rangle$ under the assumptions of the constant value of $\xi_{\text {ion }}=10^{25.2} \mathrm{erg}^{-1} \mathrm{~Hz}$, which is used in Robertson et al. (2013). The darker shade indicates the lower $\Delta \chi^{2}$. The solid and dashed lines show $68 \%$ and $95 \%$ likelihood contours, respectively.

in contrast with the claims of the pioneering study of Robertson et al. (2013) who find a parameter space of the similar analytic models explaining the observational $\tau_{e}$ and $\rho_{\mathrm{UV}}$ data available in 2013. While the best measurement value of $\tau_{e}$ is almost unchanged since then, the rapid decrease of $\rho_{\mathrm{UV}}$ at $z>8$ is clearly identified by the subsequent observational studies including our HFF work. The strong constraints on the evolution of $\rho_{\mathrm{UV}}$ at $z>8$ probably allow us to find the discrepancy between the analytic models and the observational data.

There are three possible explanations for the discrepancy between the models and the observational data of $\rho_{\mathrm{UV}}$ and $\tau_{e}$. First, the decrease of $\rho_{\mathrm{UV}}$ at $z>11$ may not be as rapid as that found at $z=8-11$. Because there is no $\rho_{\mathrm{UV}}$ data at $z>11$, in our model we extrapolate the best-fit power-law $\rho_{\mathrm{UV}}(z)$ of $z=8-11$ towards $z=30$. If the real $\rho_{\mathrm{UV}}$ values at $z>11$ are larger than this extrapolation, the $\tau_{e}$ value becomes larger, which eases the tension between the model prediction and the observations. The slow decrease of $\rho_{\mathrm{UV}}$ at $z>11$ may be made by a $M_{\text {trunc }}$ fainter than $-10 \mathrm{mag}$ and/or a luminosity function slope $(\alpha)$ steeper than the values found at $z \sim 6-8$ (Table 7 ). In other words, the discrepancy that we find may suggest that faint galaxies dominate at $z>11$ even more than at $z \sim 6-8$. It is also possible that the rapid decrease would be weakened by the 
Table 8

Best-fit parameters and the $\chi^{2}$ values

\begin{tabular}{|c|c|c|c|c|c|c|c|}
\hline & $M_{\text {trunc }}$ & $\begin{array}{c}\log \left\langle f_{\mathrm{esc}} \xi_{\text {ion }}\right\rangle \\
\left(\log \operatorname{erg}^{-1} \mathrm{~Hz}\right)\end{array}$ & $C_{\mathrm{H}_{\mathrm{II}}}$ & $\begin{array}{c}\log \rho_{\mathrm{UV}, z=8} \\
\left(\log \text { ergs s}{ }^{-1} \mathrm{~Hz}^{-1} \mathrm{Mpc}^{-3}\right)\end{array}$ & $a$ & $b$ & $\chi^{2} / \operatorname{dof}$ \\
\hline \multirow[t]{2}{*}{$\mathrm{SC}$ method } & -17 & 24.85 & 1.9 & 25.60 & 0.13 & 0.40 & $15.53 / 4$ \\
\hline & -10 & 24.38 & 1.0 & 26.11 & 0.11 & 0.09 & $11.95 / 4$ \\
\hline \multirow[t]{2}{*}{ EWC method } & -17 & 24.50 & 1.1 & 25.97 & 0.01 & 0.01 & $\ldots$ \\
\hline & -10 & 24.20 & 1.1 & 26.26 & 0.004 & 0.01 & $\cdots$ \\
\hline
\end{tabular}

luminosity function slope steepening and/or $M_{\text {trunc }}$ becoming fainter at $z \sim 9-10$. Second, the evolution of $\left\langle f_{\text {esc }} \xi_{\text {ion }}\right\rangle$ or $C_{\mathrm{H}_{\text {II }}}$ can increase $Q_{\mathrm{H}_{I I}}$, such suggested by Kuhlen \& Faucher-Giguère (2012). If the $\left\langle f_{\text {esc }} \xi_{\text {ion }}\right\rangle$ value becomes large towards high- $z, Q_{\mathrm{H}_{\mathrm{II}}}$ (accordingly $\tau_{e}$ ) could be boosted. Similarly, the small $C_{\mathrm{H}_{\mathrm{II}}}$ would enhance $\tau_{e}$, although $C_{\mathrm{H}_{\mathrm{II}}}$ can be as low as unity by definition. Third, another source of ionizing photons besides massive stars of galaxies may exist, which contributes to the cosmic reionization significantly. X-ray sources such as X-ray binaries and faint AGNs would not leave a clear signature in the $\rho_{\mathrm{UV}}$ measurements, but provide a fraction of ionizing photons via X-ray necessary for the cosmic reionization (Fragos et al. 2013; Madau et al. 2004 Mesinger et al. 2013).

The bottom right panel of Figure 17 shows $Q_{\mathrm{H}_{\mathrm{II}}}$ as a function of redshift, reproduced by our best-fit models. In Figure 17, we also plot $Q_{\mathrm{H}_{\mathrm{II}}}$ estimated from observational results of the Ly $\alpha$ forest transmission (Mesinger 2010, McGreer et al. 2011), Ly $\alpha$ near-zone sizes around high-redshift quasars (Carilli et al. 2010; Bolton et al. 2011), Ly $\alpha$ damping wing absorption in a GRB spectrum (Totani et al. 2006; McQuinn et al. 2008), evolution of the Ly $\alpha$ luminosity function and Ly $\alpha$ emitter clustering (Ota et al. 2008; Ouchi et al. 2010; Konno et al.|2014), and the Ly $\alpha$ emitting galaxy fraction evolution (Pentericci et al. 2011; Schenker et al. 2012, Ono et al. 2012). Because these measurements have uncertainties too large to constrain our model parameters, we do not use these measurements for our model fitting. However, Figure 17 illustrates that our best-fit models are in good agreement with most of the $Q_{\mathrm{H}_{\mathrm{II}}}$ measurements.

\section{SUMMARY}

We conduct the comprehensive analyses of the fulldepth HFF Abell 2744 cluster and parallel field data whose observations completed in July 2014, and study faint dropout galaxies at $z \sim 5-10$. We construct a mass model for Abell 2744 to evaluate the gravitational lensing effects of the cluster. Then we estimate number densities of our dropout candidates with realistic MonteCarlo simulations in the image plane including detection completeness, contamination, and all lensing effects such as magnification, distortion, and multiplication of images.

The major results of our study are as follows.

1. We identify 54 dropout candidates at $z \sim 5-10$ with the $i-, Y$-, and $Y J$-dropout selection criteria. The magnifications of our dropout candidates range from 1.03 to 14 . The intrinsic magnitudes of our dropout candidates reach $M_{\mathrm{UV}} \sim-17 \mathrm{mag}$ that is comparable to survey limits of the deepest blank-field observations of the HUDF.
2. The number densities of our dropout candidates are consistent with previous results of blank-field surveys. However, we find a slight excess of the number of our bright dropout candidates at $z \sim 8$ probably due to field-to-field variance.

3. We derive the UV luminosity functions at $z \sim 6-$ 7, 8, and 9 combining our HFF results with the previous blank-field surveys. We confirm that the faint-end slopes of the luminosity functions $(\alpha)$ are as steep as -2 both at $z \sim 6-7$ and $z \sim 8$. The number of dropout candidates at $z \sim 9$ increases significantly by our HFF study, and strengthen the early claim of the rapid decrease from $z \sim 8$ to $\sim 10$ from the evolution of $\rho_{\mathrm{UV}}$.

4. We use the simple analytic reionization models to explain the observational results of the $\rho_{\mathrm{UV}}$ evolution and the CMB's $\tau_{e}$. None of our models can reproduce both of these observational measurements, due to the rapid decrease of $\rho_{\mathrm{UV}}$ and the large $\tau_{e}$ value, even if we allow a large parameter space of $M_{\text {trunc }},\left\langle f_{\text {esc }} \xi_{\text {ion }}\right\rangle$, and $C_{\mathrm{H}_{\mathrm{II}}}$. This problem could be resolved by the slow decrease of $\rho_{\mathrm{UV}}$ at $z>11$, the evolution of $\left\langle f_{\mathrm{esc}} \xi_{\text {ion }}\right\rangle$ and/or $C_{\mathrm{H}_{\mathrm{II}}}$, or another source of reionization such as X-ray bright populations of X-ray binaries and faint AGN.

The HFF program will provide a significantly large sample of high-redshift galaxies when the observations of the planned six clusters are completed. In the Abell 2744 cluster field, we find 3 dropout candidates whose magnifications are $\gtrsim 10$. A simple scaling suggests that the complete HFF observations will provide $\sim 20$ highlymagnified $(\mu \gtrsim 10)$ systems at high redshift, which will uncover the properties of the faint galaxies at the epoch of cosmic reionization and greatly improve our understanding of sources of reionization up to $z \sim 12$.

We are grateful to Rychard Bouwens, Richard Ellis, Andrea Ferrara, Akio Inoue, Akira Konno, Jennifer Lotz, Kentaro Nagamine, Brant Robertson, Tomoki Saito, Takatoshi Shibuya, Dan Stark, and Masayuki Umemura for useful information, comments, and discussions. We particularly thank Hakim Atek and Rychard Bouwens for providing their data tables. This work is based on observations made with the NASA/ESA Hubble Space Telescope, obtained at the Space Telescope Science Institute (STScI), which is operated by the Association of Universities for Research in Astronomy, Inc., under NASA contract NAS 5-26555. The HST image mosaics were produced by the Frontier Fields Science Data Products Team at STScI. This work utilizes gravitational lensing models produced by P.I.s Bradač, Kneib \& Natarajan, 
Merten \& Zitrin, Sharon, and Williams, funded as part of the HST Frontier Fields program conducted by STScI. We thank these teams for their invaluable help. We are grateful to Dan Coe for the help in posting our mass model on the website. This work was supported by KAKENHI (23244025) Grant-in-Aid for Scientific Research (A) through Japan Society for the Promotion of Science (JSPS). This work was supported in part by World Premier International Research Center Initiative (WPI Initiative), MEXT, Japan, and Grant-in-Aid for Scientific Research from the JSPS (26800093). The work of M.I is partly supported by an Advanced Leading Graduate Course for Photon Science grant.

Facilities: HST (WFC3, ACS)

\section{REFERENCES}

Atek, H., Richard, J., Kneib, J.-P., et al. 2014a, ArXiv e-prints, arXiv: 1409.0512

-. 2014b, ApJ, 786, 60

Benítez, N. 2000, ApJ, 536, 571

Benitez-Llambay, A., Navarro, J. F., Abadi, M. G., et al. 2014, ArXiv e-prints, arXiv:1405.5540

Bennett, C. L., Larson, D., Weiland, J. L., et al. 2013, ApJS, 208, 20

Bertin, E., \& Arnouts, S. 1996, A\&AS, 117, 393

Bertin, E., Mellier, Y., Radovich, M., et al. 2002, in Astronomical Society of the Pacific Conference Series, Vol. 281, Astronomical Data Analysis Software and Systems XI, ed. D. A. Bohlender, D. Durand, \& T. H. Handley, 228

Bolton, J. S., Haehnelt, M. G., Warren, S. J., et al. 2011, MNRAS, 416, L70

Bouwens, R., Bradley, L., Zitrin, A., et al. 2012, ArXiv e-prints (arXiv:1211.2230), arXiv:1211.2230

Bouwens, R. J., Illingworth, G. D., Franx, M., \& Ford, H. 2007, ApJ, 670, 928

Bouwens, R. J., Illingworth, G. D., Oesch, P. A., et al. 2011, ApJ, 737, 90

—. 2014, ArXiv e-prints, arXiv:1403.4295

Bowler, R. A. A., Dunlop, J. S., McLure, R. J., et al. 2014, MNRAS, 440, 2810

Boylan-Kolchin, M., Bullock, J. S., \& Garrison-Kimmel, S. 2014, ArXiv e-prints, arXiv:1405.1040

Bradley, L. D., Trenti, M., Oesch, P. A., et al. 2012, ApJ, 760, 108

Bradley, L. D., Zitrin, A., Coe, D., et al. 2013, ArXiv e-prints, arXiv:1308.1692

Carilli, C. L., Wang, R., Fan, X., et al. 2010, ApJ, 714, 834

Coe, D., Bradley, L., \& Zitrin, A. 2014, ArXiv e-prints, arXiv:1405.0011

Dijkstra, M., Mesinger, A., \& Wyithe, J. S. B. 2011, MNRAS, 414, 2139

Ellis, R. S., McLure, R. J., Dunlop, J. S., et al. 2013, ApJ, 763, L7

Fan, X., Carilli, C. L., \& Keating, B. 2006, ARA\&A, 44, 415

Faucher-Giguère, C.-A., Kereš, D., \& Ma, C.-P. 2011, MNRAS, 417, 2982

Finkelstein, S. L., Papovich, C., Dickinson, M., et al. 2013, Nature, 502, 524

Finkelstein, S. L., Ryan, Jr., R. E., Papovich, C., et al. 2014, ArXiv e-prints, arXiv:1410.5439

Fragos, T., Lehmer, B. D., Naoz, S., Zezas, A., \& Basu-Zych, A. 2013, ApJ, 776, L31

Hinshaw, G., Larson, D., Komatsu, E., et al. 2013, ApJS, 208, 19

Jaacks, J., Thompson, R., \& Nagamine, K. 2013, ApJ, 766, 94

Jaffe, W. 1983, MNRAS, 202, 995

Jauzac, M., Richard, J., Jullo, E., et al. 2014, ArXiv e-prints, arXiv: 1409.8663

Johnson, T. L., Sharon, K., Bayliss, M. B., et al. 2014, ArXiv e-prints (arXiv:1405.0222), arXiv:1405.0222

Kashikawa, N., Shimasaku, K., Malkan, M. A., et al. 2006, ApJ, 648,7

Kashikawa, N., Shimasaku, K., Matsuda, Y., et al. 2011, ApJ, 734,119

Keeton, C. R. 2001, ArXiv Astrophysics e-prints (astro-ph/0102341), astro-ph/0102341
Kochanek, C. S. 1991, ApJ, 373, 354

Konno, A., Ouchi, M., Ono, Y., et al. 2014, ArXiv e-prints, arXiv:1404.6066

Kron, R. G. 1980, ApJS, 43, 305

Kuhlen, M., \& Faucher-Giguère, C.-A. 2012, MNRAS, 423, 862

Lam, D., Broadhurst, T., Diego, J. M., et al. 2014, ArXiv e-prints, arXiv:1406.2702

Laporte, N., Streblyanska, A., Clement, B., et al. 2014, A\&A, $562, \mathrm{~L} 8$

Madau, P., Ferguson, H. C., Dickinson, M. E., et al. 1996, MNRAS, 283, 1388

Madau, P., Pozzetti, L., \& Dickinson, M. 1998, ApJ, 498, 106

Madau, P., Rees, M. J., Volonteri, M., Haardt, F., \& Oh, S. P. 2004, ApJ, 604, 484

McGreer, I. D., Mesinger, A., \& Fan, X. 2011, MNRAS, 415, 3237

McLure, R. J., Dunlop, J. S., Bowler, R. A. A., et al. 2013, MNRAS, 432, 2696

McQuinn, M., Hernquist, L., Zaldarriaga, M., \& Dutta, S. 2007, MNRAS, 381, 75

McQuinn, M., Lidz, A., Zaldarriaga, M., Hernquist, L., \& Dutta, S. 2008, MNRAS, 388, 1101

Mesinger, A. 2010, MNRAS, 407, 1328

Mesinger, A., Ferrara, A., \& Spiegel, D. S. 2013, MNRAS, 431, 621

Mesinger, A., \& Furlanetto, S. R. 2008, MNRAS, 386, 1990

Meurer, G. R., Heckman, T. M., \& Calzetti, D. 1999, ApJ, 521, 64

Montes, M., \& Trujillo, I. 2014, ArXiv e-prints, arXiv:1405.2070

Nakamura, T. T., \& Suto, Y. 1997, Progress of Theoretical Physics, 97, 49

Navarro, J. F., Frenk, C. S., \& White, S. D. M. 1997, ApJ, 490, 493

Oesch, P. A., Bouwens, R. J., Illingworth, G. D., et al. 2014, ArXiv e-prints, arXiv:1409.1228

Oesch, P. A., Bouwens, R. J., Carollo, C. M., et al. 2010, ApJ, 709, L21

Oesch, P. A., Bouwens, R. J., Illingworth, G. D., et al. 2013, ApJ, 773,75

Oguri, M. 2010, PASJ, 62, 1017

Ono, Y., Ouchi, M., Mobasher, B., et al. 2012, ApJ, 744, 83

Ono, Y., Ouchi, M., Curtis-Lake, E., et al. 2013, ApJ, 777, 155

Ota, K., Iye, M., Kashikawa, N., et al. 2008, ApJ, 677, 12

Ouchi, M., Shimasaku, K., Okamura, S., et al. 2004, ApJ, 611, 660

Ouchi, M., Mobasher, B., Shimasaku, K., et al. 2009, ApJ, 706, 1136

Ouchi, M., Shimasaku, K., Furusawa, H., et al. 2010, ApJ, 723, 869

Owers, M. S., Randall, S. W., Nulsen, P. E. J., et al. 2011, ApJ, 728, 27

Pentericci, L., Fontana, A., Vanzella, E., et al. 2011, ApJ, 743, 132

Pentericci, L., Vanzella, E., Fontana, A., et al. 2014, ArXiv e-prints, arXiv:1403.5466

Planck Collaboration, Ade, P. A. R., Aghanim, N., et al. 2013, ArXiv e-prints, arXiv:1303.5076

Ravindranath, S., Giavalisco, M., Ferguson, H. C., et al. 2006, ApJ, 652, 963

Reichardt, C. L., Shaw, L., Zahn, O., et al. 2012, ApJ, 755, 70

Richard, J., Jauzac, M., Limousin, M., et al. 2014, ArXiv e-prints, arXiv: 1405.3303

Robertson, B. E., Ellis, R. S., Dunlop, J. S., McLure, R. J., \& Stark, D. P. 2010, Nature, 468, 49

Robertson, B. E., Ellis, R. S., Dunlop, J. S., et al. 2014, ArXiv e-prints, arXiv:1410.0962

Robertson, B. E., Furlanetto, S. R., Schneider, E., et al. 2013, ApJ, 768, 71

Schenker, M. A., Ellis, R. S., Konidaris, N. P., \& Stark, D. P. 2014, ArXiv e-prints, arXiv: 1404.4632

Schenker, M. A., Stark, D. P., Ellis, R. S., et al. 2012, ApJ, 744, 179

Schenker, M. A., Robertson, B. E., Ellis, R. S., et al. 2013, ApJ, 768,196

Story, K. T., Reichardt, C. L., Hou, Z., et al. 2013, ApJ, 779, 86

Tody, D. 1986, in Society of Photo-Optical Instrumentation Engineers (SPIE) Conference Series, Vol. 627, Instrumentation in astronomy VI, ed. D. L. Crawford, 733 
Tody, D. 1993, in Astronomical Society of the Pacific Conference Series, Vol. 52, Astronomical Data Analysis Software and Systems II, ed. R. J. Hanisch, R. J. V. Brissenden, \& J. Barnes, 173

Totani, T., Kawai, N., Kosugi, G., et al. 2006, PASJ, 58, 485

Totani, T., Aoki, K., Hattori, T., et al. 2013, ArXiv e-prints, arXiv:1312.3934
Treu, T., Schmidt, K. B., Trenti, M., Bradley, L. D., \& Stiavelli, M. 2013, ApJ, 775, L29

Weisz, D. R., Dolphin, A. E., Skillman, E. D., et al. 2014, ArXiv e-prints, arXiv:1405.3281

Yagi, M., Kashikawa, N., Sekiguchi, M., et al. 2002, AJ, 123, 66 Zheng, W., Shu, X., . Moustakas, J., et al. 2014, ArXiv e-prints (arXiv:1402.6743), arXiv:1402.6743

Zitrin, A., Zheng, W., Broadhurst, T., et al. 2014, ArXiv e-prints, arXiv:1407.3769 